\title{
Role of chronic ryanodine receptor phosphorylation in heart failure and $\beta$-adrenergic receptor blockade in mice
}

\author{
Jian Shan, ${ }^{1}$ Matthew J. Betzenhauser, ${ }^{1}$ Alexander Kushnir, ${ }^{1}$ Steven Reiken, ${ }^{1}$ \\ Albano C. Meli, ${ }^{1}$ Anetta Wronska, ${ }^{1}$ Miroslav Dura, ${ }^{1}$ Bi-Xing Chen, ${ }^{1}$ and Andrew R. Marks ${ }^{1,2}$ \\ ${ }^{1}$ Clyde and Helen Wu Center for Molecular Cardiology, Department of Physiology and Cellular Biophysics, and \\ 2Department of Medicine, College of Physicians and Surgeons of Columbia University, New York, New York, USA.
}

\begin{abstract}
Increased sarcoplasmic reticulum (SR) $\mathrm{Ca}^{2+}$ leak via the cardiac ryanodine receptor/calcium release channel (RyR2) is thought to play a role in heart failure (HF) progression. Inhibition of this leak is an emerging therapeutic strategy. To explore the role of chronic PKA phosphorylation of RyR2 in HF pathogenesis and treatment, we generated a knockin mouse with aspartic acid replacing serine 2808 (mice are referred to herein as RyR2$\mathrm{S}^{2808 \mathrm{D}^{+/+}}$mice). This mutation mimics constitutive PKA hyperphosphorylation of RyR2, which causes depletion of the stabilizing subunit FKBP12.6 (also known as calstabin2), resulting in leaky RyR2. RyR2-S2808D ${ }^{+/+}$ mice developed age-dependent cardiomyopathy, elevated RyR2 oxidation and nitrosylation, reduced SR $\mathrm{Ca}^{2+}$ store content, and increased diastolic SR Ca ${ }^{2+}$ leak. After myocardial infarction, RyR2-S2808D ${ }^{+/+}$mice exhibited increased mortality compared with WT littermates. Treatment with S107, a 1,4-benzothiazepine derivative that stabilizes RyR2-calstabin2 interactions, inhibited the RyR2-mediated diastolic SR $\mathrm{Ca}^{2+}$ leak and reduced $\mathrm{HF}$ progression in WT and RyR2-S2808D ${ }^{+/+}$mice. In contrast, $\beta$-adrenergic receptor blockers improved cardiac function in WT but not in RyR2-S2808D ${ }^{+/+}$mice.Thus, chronic PKA hyperphosphorylation of RyR2 results in a diastolic leak that causes cardiac dysfunction. Reversing PKA hyperphosphorylation of RyR2 is an important mechanism underlying the therapeutic action of $\beta$-blocker therapy in HF.
\end{abstract}

\section{Introduction}

Heart failure (HF), a syndrome characterized by progressive deterioration of cardiac function, represents a major public health burden and is a leading cause of morbidity and mortality in the Western world, with more than 5 million affected Americans (1). HF treatment includes administration of $\beta$-adrenergic receptor ( $\beta$-AR) blockers ( $\beta$-blockers), which improve cardiac function and reduce mortality (2-4). We have previously reported that $\beta$-blocker therapy inhibits PKA hyperphosphorylation and remodeling of the RyR2 channel complex in humans and animals with $\operatorname{HF}(5,6)$. While treatment with $\beta$-blockers represents a major therapeutic strategy for $\mathrm{HF}$ patients, the precise mechanistic basis of action of these drugs in $\mathrm{HF}$ downstream of $\beta$-AR blockade remains poorly understood.

During cardiac excitation-contraction coupling, an action potential triggers a small amount of $\mathrm{Ca}^{2+}$ to enter the myocyte through the L-type $\mathrm{Ca}^{2+}$ channel (LTCC, Cav1.2). This event triggers a much larger intracellular $\mathrm{Ca}^{2+}$ release by activating the ryanodine receptor/calcium release channel (RyR2) on the sarcoplasmic reticulum (SR), a phenomenon referred to as calcium-induced calcium release $(7,8) . \mathrm{Ca}^{2+}$ released from the $\mathrm{SR}$ into the cytosol binds to troponin $\mathrm{C}$, causing a conformational change that enables actinmyosin cross bridging to occur, resulting in shortening of the sarcomere and cardiac contraction $(9,10) . \mathrm{Ca}^{2+}$ reuptake into the $\mathrm{SR}$ via the $\mathrm{Ca}^{2+}$-ATPase and removal from the cell, mainly by the

Authorship note: Jian Shan, Matthew J. Betzenhauser, Alexander Kushnir, and Steven Reiken contributed equally to this work.

Conflict of interest: Andrew R. Marks is a consultant for a start-up company, ARMGO Pharma Inc., that is targeting RyR2 to prevent heart failure and sudden cardiac death.

Citation for this article: J Clin Invest. 2010;120(12):4375-4387. doi:10.1172/JCI37649.
$\mathrm{Na}^{+} / \mathrm{Ca}^{2+}$ exchanger, lowers $\left[\mathrm{Ca}^{2+}\right]_{c y t}$, resulting in dissociation of $\mathrm{Ca}^{2+}$ from troponin $\mathrm{C}$ and cardiac relaxation. Disruption of this highly coordinated process can trigger arrhythmias, and defects in $\mathrm{Ca}^{2+}$ cycling are associated with HF progression (11).

$\mathrm{HF}$ is characterized by a sustained hyperadrenergic state in which serum catecholamine levels are chronically elevated (12). We have shown that one consequence of this chronic hyperadrenergic state is persistent PKA hyperphosphorylation of RyR2, such that 3-4 out of the 4 Ser2808 in each homotetrameric RyR2 channel are chronically phosphorylated (13). We have shown that chronic PKA hyperphosphorylation of RyR2 is associated with depletion of the channel-stabilization subunit calstabin2 from the RyR2 channel macromolecular complex. This results in a diastolic SR $\mathrm{Ca}^{2+}$ leak that depletes the SR of $\mathrm{Ca}^{2+}$ contributing to impaired contractility (13) and is associated with fatal cardiac arrhythmias due to activation of inward depolarizing currents (14). Indeed, mice engineered with RyR2 lacking the PKA phosphorylation site are protected from HF progression after myocardial infarction (MI) (15). Furthermore, we have shown that the chronic PKA hyperphosphorylation of RyR2 in HF is associated with remodeling of the RyR2 macromolecular complex that includes depletion of phosphatases (13) PP1 and PP2a (impairing dephosphorylation of Ser2808) and the cAMP-specific type 4 phosphodiesterase (PDE4D3) from the RyR2 complex. Depletion of PDE4D3 from the RyR2 complex causes sustained elevation of local cAMP levels (16). There is general agreement with the key finding of our studies, which is that diastolic SR $\mathrm{Ca}^{2+}$ leak contributes to HF progression and arrhythmias (17). However, several specific aspects of our model are controversial, including the role of RyR2 PKA hyperphosphorylation and calstabin 2 depletion in HF. These findings have both been confirmed (18-26) and challenged in the literature (27-31). 


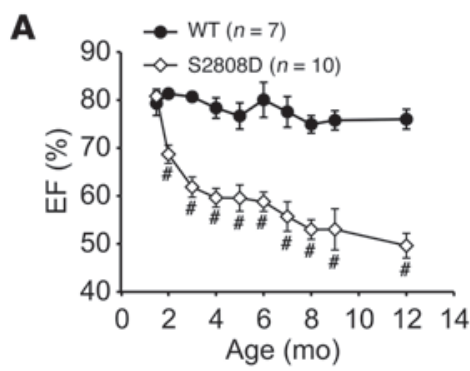

D

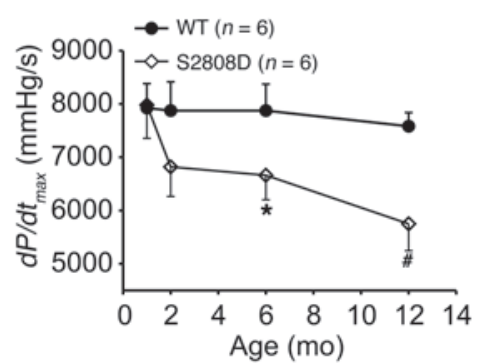

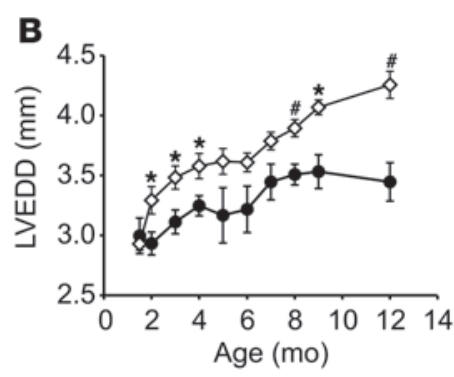

E

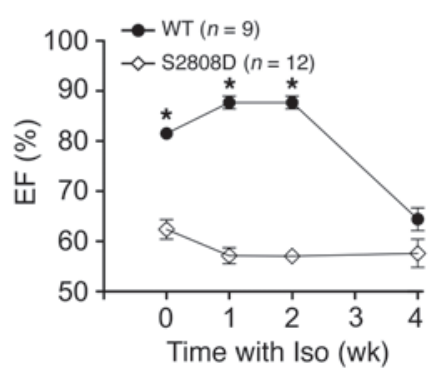

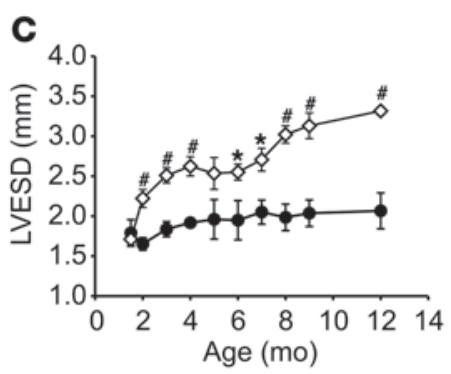

$\mathbf{F}$

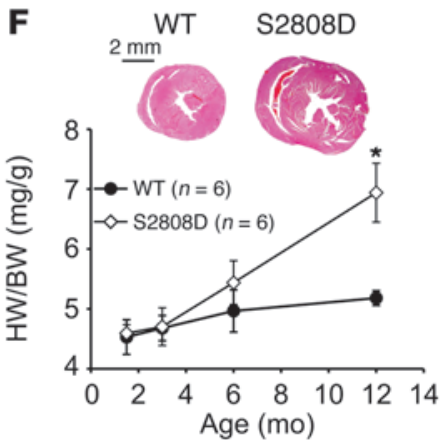

\section{Figure 1}

Age-dependent cardiomyopathy in RyR2-S2808D+/+ mice. (A-C) Serial echocardiographic measurements were performed from 2 to 12 months of age in RyR2-S2808D+/+ (S2808D) mice and WT littermates. Compared with WT littermates, RyR2-S2808D+/+ mice exhibited progressive cardiac dysfunction and LV enlargement. LVEDD, LV end-diastolic diameter (WT, $n=7$; RyR2-S2808D ${ }^{+/+}, n=10$; $\#<0.01$ versus WT; * $P<0.05$ versus WT). (D) Cardiac catheterization also revealed age-dependent cardiac dysfunction $\left({ }^{\star} P<0.05\right.$ versus WT; ${ }^{*} P<0.01$ versus WT). (E) Chronic Iso treatment in RyR2-S2808D+/+ mice and WT littermates. At 4 months of age, RyR2-S2808D +/+ and WT mice were treated with Iso (30 mg/kg/d) for 4 weeks. Cardiac function was monitored with echocardiography at baseline, 1 , 2, and 4 weeks ( ${ }^{*} P<0.05$ versus RyR2-S2808D ${ }^{++}$mice). (F) Representative histology of age-matched RyR2-S2808D+/+ and WT littermates (top). Cross section at papillary muscle level. Scale bar: $2 \mathrm{~mm}$. Heart weight to body weight (HW/BW) ratio (bottom). Filled circles represent WT mice; open diamonds represent RyR2-S2808D ${ }^{+/+}$mice $\left({ }^{\star} P<0.05\right.$ versus WT).

Given the complexity of PKA signaling in the heart, it is difficult to examine the effects of chronic PKA hyperphosphorylation of $\mathrm{RyR} 2$ in the absence of effects on other $\mathrm{Ca}^{2+}$-handling proteins. In order to overcome these difficulties, we generated a knockin mouse model that mimics constitutive PKA hyperphosphorylation of RyR2, in which the Ser2808 in RyR2 was substituted with an aspartic acid residue (herein referred to as RyR2-S2808D mice). In contrast to our earlier results using recombinant human RyR2-S2809D channels, RyR2 from young RyR2-S2808D mice ( $\leq 3$ months of age) were able to bind calstabin2 and displayed normal single-channel activity. However, older RyR2-S2808D mice (> 6 months of age) exhibited decreased calstabin 2 binding to RyR 2 and decreased cardiac function, which correlated with dysfunctional RyR2-S2808D channels examined in planar lipid bilayers and increased diastolic $\mathrm{SR} \mathrm{Ca}^{2+}$ leak in ventricular myocytes. These effects were associated with progressive oxidation and nitrosylation of RyR2 in RyR2S2808D mice, suggesting that chronic PKA hyperphosphorylation, combined with oxidation and nitrosylation, substantially disrupts channel activity in vivo. Furthermore, $\beta$-blockers improved cardiac function in WT mice after MI but failed to improve cardiac function in RyR2-S2808D $\mathrm{D}^{+/}$mice after MI, suggesting that inhibition of PKA phosphorylation of RyR2 at Ser2808 is a critical mechanism by which $\beta$-blockers improve cardiac function in HF.

\section{Results}

Generation of the RyR2-S2808D knockin mouse model. The genotypes of ES cells and knockin mice were confirmed using both Southern blotting and PCR (Supplemental Figure 1 and Supplemental
Methods; supplemental material available online with this article; doi:10.1172/JCI37649DS1). RyR2-S2808D mice were viable and fertile and were born at the expected Mendelian frequencies (WT/WT, $n=58,25 \%$; WT/RyR2-S2808D, $n=116$, 49\%; RyR2-S2808D/RyR2S2808D, $n=62,26 \%$ ).

Mimicking chronic PKA phosphorylation of RyR2 causes age-dependent cardiomyopathy. Homozygous RyR2-S2808D (RyR2-S2808D ${ }^{+/+}$) and WT littermates were examined using echocardiography and cardiac catheterization from 1 to 12 months of age to determine the consequences of chronic PKA phosphorylation of RyR2 on cardiac function. Serial echocardiographic measurements over the course of a year revealed that RyR2-S2808D ${ }^{+/+}$mice exhibited progressive, age-dependent cardiac dysfunction. Cardiac dysfunction was manifested as a reduction in the ejection fraction (EF) coupled with a slight increase in LV diameter (Figure 1, A-C, and Supplemental Table 1). Similarly, cardiac catheterization at 1, 2, 6, and 12 months of age revealed a progressive deterioration of cardiac contractility in RyR2-S2808D $\mathrm{D}^{+/}$mice that was not evident in WT littermates (Figure 1D). This reduction in cardiac function was comparable to the effects of chronic isoproterenol (Iso) treatment of 4-monthold WT mice compared with that of age-matched RyR2-S2808D $+/+$ mice (Figure 1E). At 4 months of age, there was already a significant reduction in EF in the RyR2-S2808D ${ }^{+/+}$mice ( $\left.60 \%\right)$. Chronic Iso treatment did not cause a further reduction in $\mathrm{EF}$, whereas it reduced EF in the WT mice, such that after 4 weeks of Iso treatment, the EF of the WT mice was reduced from approximately $80 \%$ to a level ( $65 \%)$ comparable with that of the RyR2-S2808D ${ }^{+/+}$ mice (Figure 1E). However, chronic Iso infusion did cause compa- 

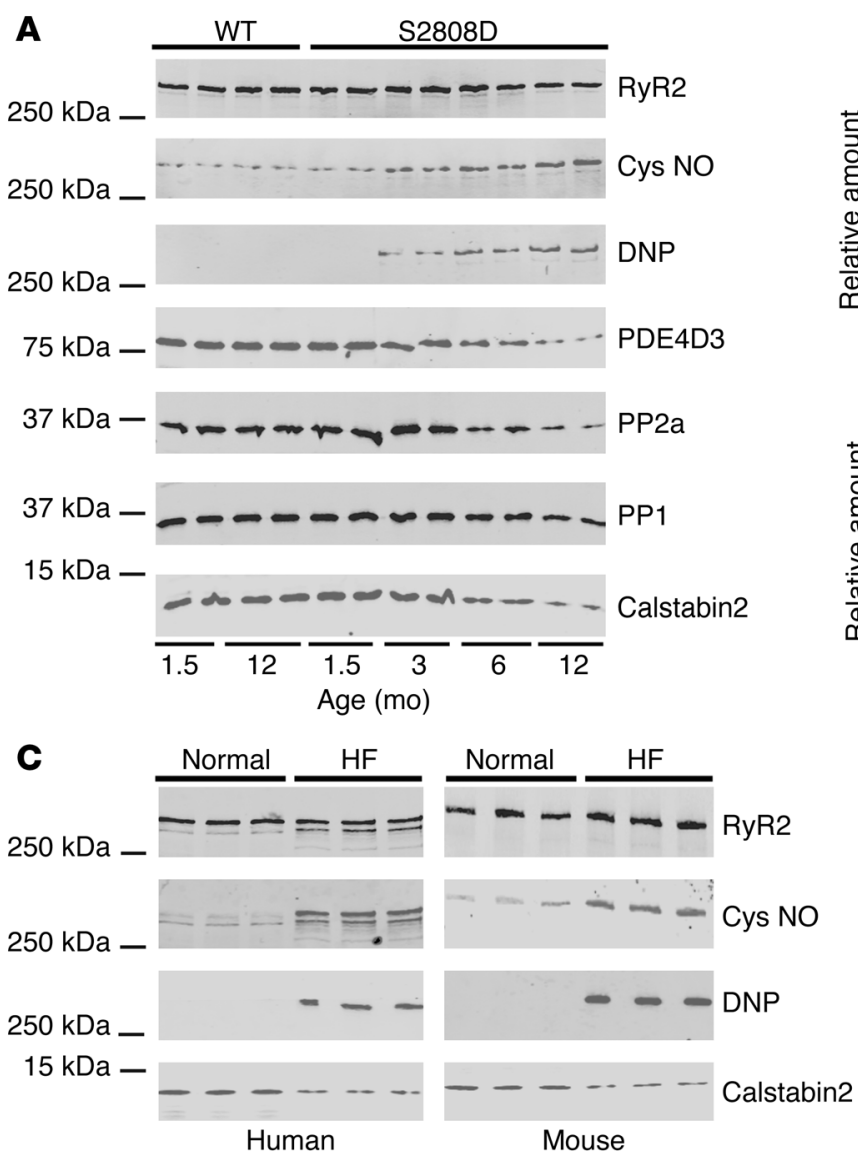

B
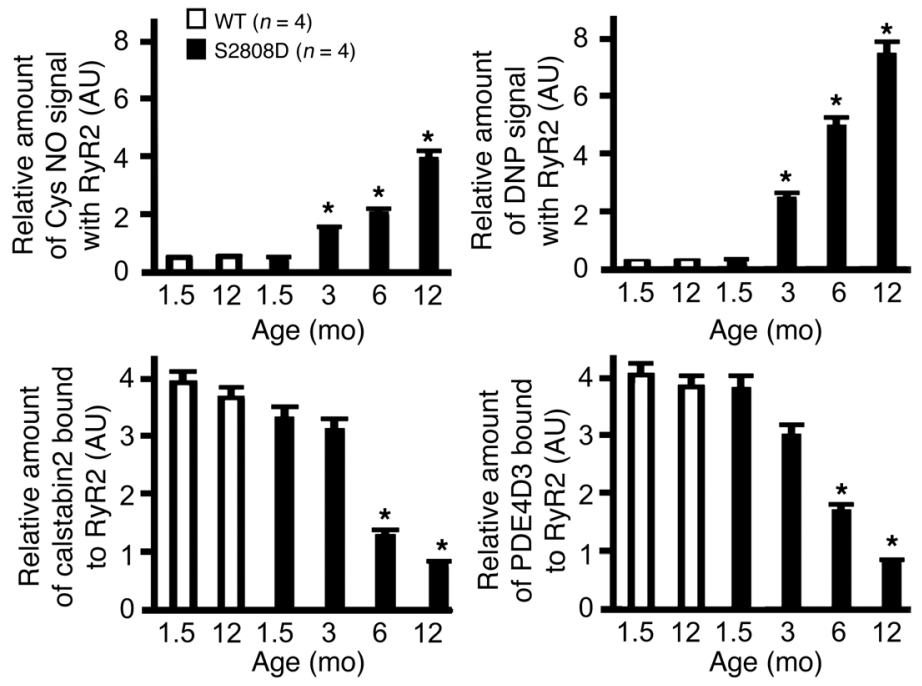

D

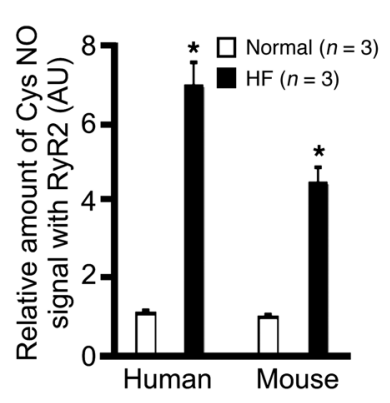

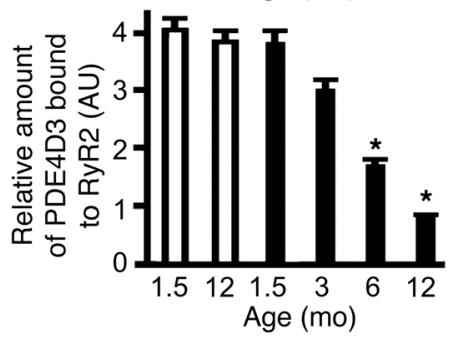

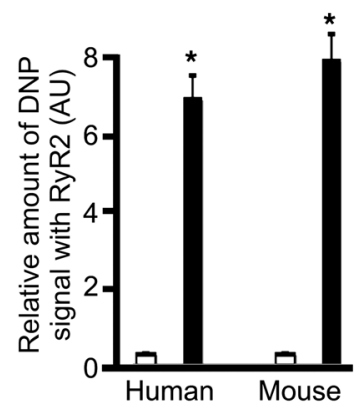

Figure 2

RyR2 channel complex remodeling in RyR2-S2808D mice. (A) Progressive oxidation, Cys-nitrosylation (Cys NO) of cardiac RyR2, and depletion of PDE4D3, calstabin2, PP2A, and PP1 from the cardiac RyR2 complex in the RyR2-S2808D mice. (B) Levels of proteins in the RyR2 complex and levels of oxidation and Cys-nitrosylation were normalized to the total amount of RyR2 (AU). ${ }^{*} P<0.05$ versus WT at 1.5 months. (C) Oxidation and Cys-nitrosylation of RyR2 in HF samples. RyR2 immunoprecipitations were performed on hearts from mice with MI and sham-treated mice or on samples from HF and non-HF human heart tissue. (D) Levels of oxidation and Cys-nitrosylation were normalized to the total amount of RyR2 (AU). * $P<0.05$ versus normal samples.

rable increases in LV end-systolic diameter (LVESD) in the WT and S2808D mice (Supplemental Table 2). These results are consistent with our findings in the companion manuscript in which cardiac function in the RyR2-S2808A mice was similarly protected from the deleterious effects of chronic Iso (32); however, chronic Iso infusion did result in an increase in LVESD (30).

RyR2-S2808D ${ }^{+/+}$mice also exhibited increased heart weight to body weight ratios compared with those of WT controls (Figure 1F). Histological studies of RyR2-S2808D ${ }^{+/+}$hearts at 2, 6, and 12 months of age confirmed the results of echocardiography, showing that hearts from RyR2-S2808D $\mathrm{D}^{+/+}$mice exhibited mild enlargement at 12 months of age. No increased fibrosis was detected using Masson's trichrome staining compared with WT littermates (Supplemental Figure 2, A-D). In addition, apoptosis assessed by TUNEL staining showed no differences between WT and RyR2-S2808D +/+ mice at any age examined (Supplemental Figure 2, E and F).

Age-dependent oxidation and nitrosylation of RyR2 in RyR2-S2808D mice. We have reported that the RyR2 macromolecular complex undergoes remodeling during $\mathrm{HF}$ (13), characterized by depletion of calstabin2, PDE4D3, and the phosphatases PP1 and PP2A from the RyR2 macromolecular complex $(13,16)$. In order to determine whether the age-dependent reduction in cardiac function observed in RyR2-S2808D $\mathrm{D}^{+/+}$mice (Figure 1) was associated with RyR2 complex remodelling, components of the RyR2 macromolecular complex, including PP1 and PP2A, PDE4D3, and calstabin2, were examined using coimmunoprecipitation with an $\alpha$-RyR2 antibody. There were significant decreases in the levels of PDE4D3, PP1, PP2A, and calstabin2 in the RyR2 complex by 6 to 12 months of age, consistent with development of cardiac dysfunction (Figure 2A) and comparable with the remodeling of the RyR2 complex that we have shown occurs in failing hearts $(13,16)$. The levels of RyR2 and calstabin 2 in cardiomyocytes were not changed with age in the WT or RyR2-S2808D $\mathrm{D}^{+/}$mice at the time points examined (Supplemental Figure 3).

There was a progressive, age-dependent depletion of calstabin2 from the RyR2 complex, with minimal ( 20\%) calstabin2 depletion in 6-week-old RyR2-S2808 $\mathrm{D}^{+/+}$mice and near complete depletion of calstabin2 from the RyR2 complex in 12-month-old RyR2-S2808D ${ }^{+/+}$mice (Figure $2 \mathrm{~A}$ ). The progressive, age-dependent depletion of calstabin2 from the RyR2-S2808D ${ }^{+/+}$complex cannot be explained by progressive PKA hyperphosphorylation of the channel, since the PKA site, Ser2808, is not available to be 
A
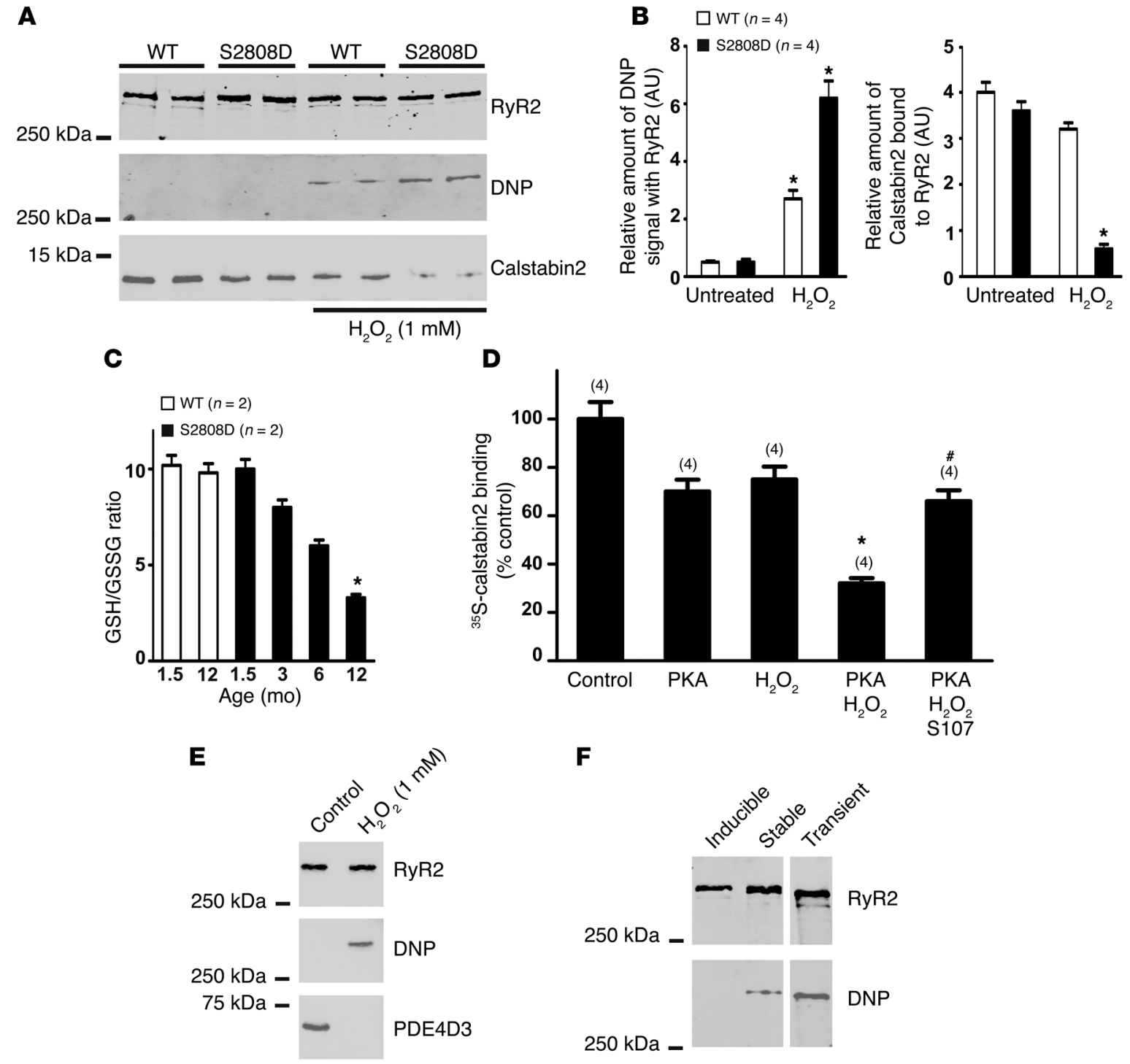

Figure 3

Combined effects of PKA phosphorylation and oxidation on calstabin2 binding to RyR2. (A) CSR preparations were treated with $1 \mathrm{mM} \mathrm{H}_{2} \mathrm{O}_{2}$, and RyR2 was immunoprecipitated, size fractionated, and immunoblotted for oxidation (DNP) and calstabin2 in the RyR2 complex. (B) Levels of oxidation and calstabin2 in the RyR2 complex were normalized to the total amount of RyR2 (AU). ${ }^{*} P<0.05$ untreated versus $\mathrm{H}_{2} \mathrm{O}_{2}$-treated samples. (C) GSH/GSSG ratios $(n=2)$ were compared between WT and RyR2-S2808D+/+ mice. ${ }^{*} P<0.05$. (D) ${ }^{35}$ S-calstabin binding was measured in samples treated with $\mathrm{PKA}, \mathrm{H}_{2} \mathrm{O}_{2}$, or a combination of the 2 , in the presence or absence or S107. Radioactivity counts were normalized to the untreated control samples. Data are presented as mean \pm SEM $(n=4)$. The numbers of replicates for each condition are indicated by the parenthetical numbers over each bar. ${ }^{*} P<0.05$ compared with control; ${ }^{*} P<0.05$ compared with $\mathrm{PKA} / \mathrm{H}_{2} \mathrm{O}_{2}$ treatment without $\mathrm{S} 107$. (E) CSR was treated with $1 \mathrm{mM} \mathrm{H}_{2} \mathrm{O}_{2}$, and RyR2 was immunoprecipitated and immunoblotted for oxidation (DNP) and PDE4D3 in the RyR2 complex. (F) RyR2 was expressed in $\mathrm{CHO}$ cells using an inducible vector (tetracycline), transient transfection, or stable transfection and immunoblotted with anti-DNP antibody to determine oxidation of the channel.

phosphorylated in the RyR2-S2808D $\mathrm{D}^{+/}$channels. We therefore examined oxidation of RyR2, since we have recently observed that oxidation of RyR2 enhances PKA phosphorylation-induced depletion of calstabin 2 from the RyR2 complex (see companion manuscript; ref. 32). Furthermore, oxidizing agents have been shown to promote RyR2-mediated SR $\mathrm{Ca}^{2+}$ leak, and RyR2 was reported to be oxidized in failing hearts from a canine model of $\operatorname{HF}(33,34)$. We also examined Cys-nitrosylation of RyR2, since Cys-nitrosylation has been reported to cause depletion of calsta- bin 1 from the RyR1 complex in skeletal muscle $(35,36)$ and we have recently shown that Cys-nitrosylation of RyR 1 and RyR 2 in skeletal and cardiac muscles from a mouse model of Duchenne muscular dystrophy causes depletion of calstabin 1 and calstabin2 from RyR1 and RyR2s, respectively $(36,37)$. Indeed, there were age-dependent increases in both Cys-nitrosylation and oxidation of cardiac RyR2 from RyR2-S2808D mice (Figure 2B) that likely contribute to the age-dependent depletion of calstabin2 from the RyR2 complex in RyR2-S2808D mice. 
A $\quad$ WT $(1.5 \mathrm{mo})$

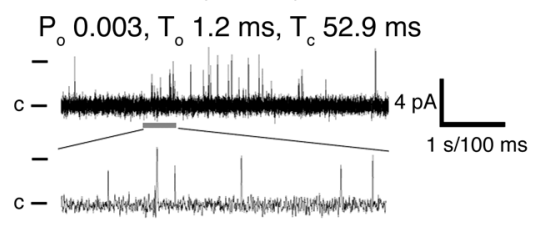

C $\quad \mathrm{WT}(10 \mathrm{mo})$

$P_{0} 0.023, T_{0} 8.2 \mathrm{~ms}, T_{c} 36.7 \mathrm{~ms}$

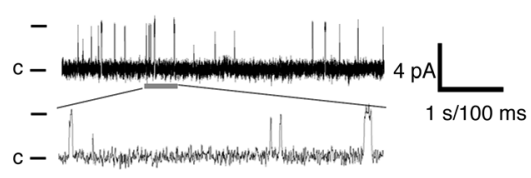

S2808D (1.5 mo)

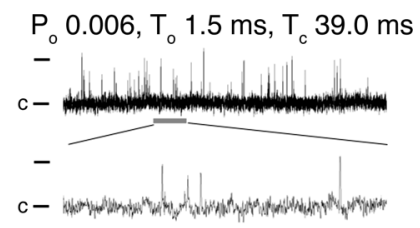

$\mathrm{S} 2808 \mathrm{D}(10 \mathrm{mo})$

$P_{0} 0.411, T_{0} 13.9 \mathrm{~ms}, T_{c} 8.1 \mathrm{~ms}$

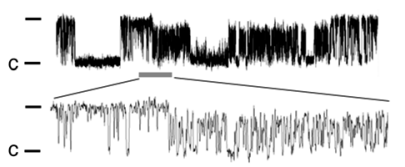

B

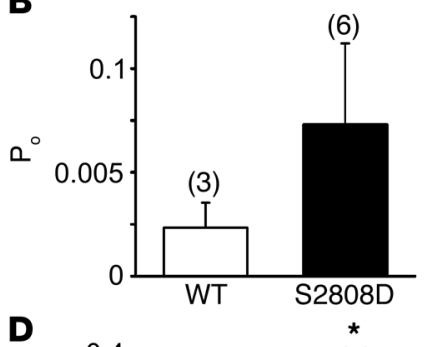

D

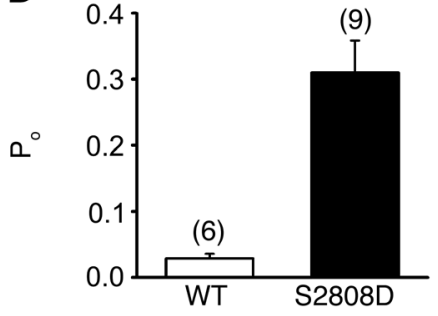

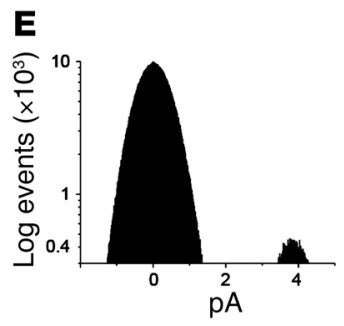
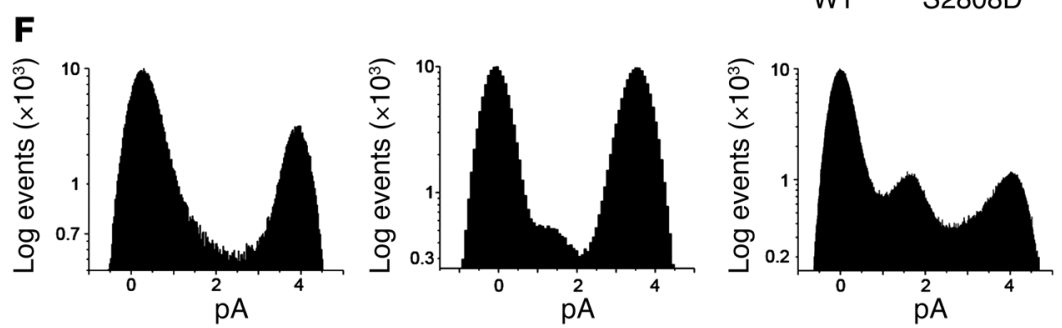

Figure 4

Functional characterization of cardiac RyR2 channels from WT and RyR2-S2808D+/+ mice. (A) Representative single channel current traces of cardiac RyR2 channels isolated from WT and RyR2-S2808D+/+ mice at 1.5 months of age. (B) Bar graph summarizing average Po in WT $(n=3)$ and RyR2-S2808 $\mathrm{D}^{+/+}(n=6)$ channels from 1.5-month-old mice $(P=\mathrm{NS})$. The number of channels recorded from each sample is indicated by the parenthetical numbers over each bar. (C) Representative single channel current traces of cardiac RyR2 channels isolated from 10-month-old WT and RyR2-S2808D ${ }^{+/+}$mice. (D) Bar graph summarizing average Po in WT $(n=6)$ and S2808D ${ }^{+/+}(n=9)$ channels from 10-month-old mice $\left({ }^{\star} P<0.05\right)$. The number of channels recorded from each sample is indicated by the parenthetical numbers over each bar. Channel openings are shown as upward deflections; the open and closed (c) states of the channel are indicated by horizontal bars in the beginning of each trace. Examples of the channel activity are shown at 2 different time scales ( $5 \mathrm{~s}$ for upper trace and $500 \mathrm{~ms}$ for lower trace depicted by the thick gray bar) as indicated by dimension bars. The respective Po, To (average open time), and Tc (average closed time) are shown above each 5 second trace and correspond to that particular experiment. (E) Amplitude histogram of a representative WT cardiac RyR2 channel (at 10 months old), showing 2 distinct peaks corresponding to fully open $(\sim 4 p A)$ and closed (0pA) states of the channel. (F) Samples of amplitude histograms from 3 different experiments, using RyR2-S2808D+/+ channels (10-month-old mice), showing subconductance states.

To determine whether increased Cys-nitrosylation and oxidation of RyR 2 occur in failing hearts, we examined RyR2 from 3 patients with end-stage HF and from mice with post-MI HF (Figure 2C). RyR2 was nitrosylated and oxidized in both human and murine failing hearts (Figure 2, C and D).

We next assessed the effects of treating cardiac microsomal fractions with $\mathrm{H}_{2} \mathrm{O}_{2}$. $\mathrm{H}_{2} \mathrm{O}_{2}(1 \mathrm{mM})$ resulted in oxidation of RyR2 (determined by assessment of DNP-derivatized RyR2) and a reduction in the amount of calstabin2 in the RyR2 complex in cardiac microsomes from WT mice by approximately $20 \%$ (similar to an earlier report; ref. 38). In contrast, oxidation of RyR2-S2808D (which mimics constitutively PKA-phosphorylated channels) by $1 \mathrm{mM}$ $\mathrm{H}_{2} \mathrm{O}_{2}$ caused nearly complete depletion of calstabin2 from the RyR2 complex in cardiac microsomes from 1.5-month-old RyR2S2808D mice (Figure 3, A and B).

To assess oxidative/nitrosative stress in the hearts of RyR2S2808D mice, we measured the levels of the primary intracellular antioxidant buffer glutathione (GSH); its oxidized form GSH disulfide (GSSG); and the GSH/GSSG ratios in 1.5- to 12-month-old mice. By 12 months of age, RyR2-S2808D hearts showed significant basal oxidative stress, with an approximately $70 \%$ reduction in the GSH/GSSG ratio (Figure 3C), reflecting changes in ROS and/or RNS levels.

In order to quantify the effects of oxidation and PKA phosphorylation of RyR2 on calstabin 2 binding to the channel, we used a ${ }^{35} \mathrm{~S}$-calstabin 2 binding assay (see Methods). PKA phosphorylation of RyR2 reduced calstabin2 binding to RyR2 by approximately $30 \%$. Oxidation of RyR 2 with $1 \mathrm{mM} \mathrm{H}_{2} \mathrm{O}_{2}$ reduced calstabin 2 binding to RyR 2 by approximately $20 \%$. The combination of PKA phosphorylation and oxidation of RyR2 reduced calstabin 2 binding to RyR 2 by approximately $70 \%(P<0.01$ compared with control, $n=4$ ) (Figure 3D).

We have previously shown that JTV-519, a 1,4-benzothiazepine, inhibits depletion of calstabin2 from PKA-hyperphosphorylated RyR2 channels and reduces HF progression and ventricular arrhythmias by inhibiting diastolic SR $\mathrm{Ca}^{2+}$ leak via RyR2 $(16,39,40)$. Moreover, these effects of JTV-519 (K201) were not observed in casltabin2-deficient mice, demonstrating the importance of calstabin2 in the mechanism of action of this class of drugs (39). In addition to acting on the RyR2 channel to prevent depletion 
A

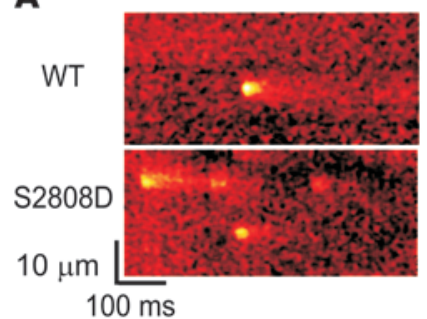

D

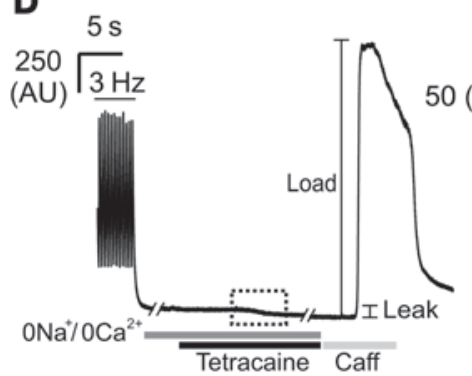

B

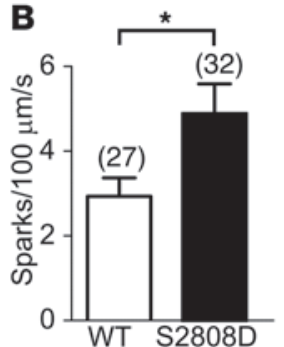

E

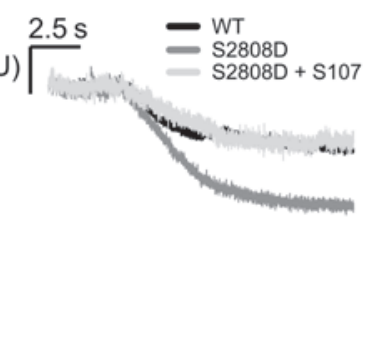

C
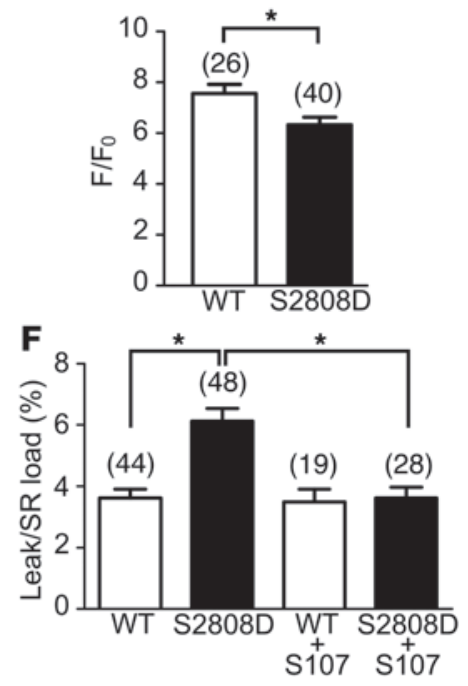

\section{Figure 5}

Reduced store content and increased diastolic $\mathrm{Ca}^{2+}$ leak in RyR2-S2808D+/+ mice. (A) Representative line scans obtained from ventricular myocytes isolated from WT and RyR2-S2808D+/+ mice, showing an increased spark frequency in RyR2-S2808D myocytes. (B) Pooled data showing mean \pm SEM spark frequency from the number of myocytes indicated parenthetically. ${ }^{*} P<0.05$. (C) Pooled data showing mean \pm SEM amplitude of caffeine-evoked signals from the number of cells indicated parenthetically. ${ }^{*} P<0.05$. (D) Representative trace depicting the protocol for determining the level of SR $\mathrm{Ca}^{2+}$ leak in ventricular myocytes. After termination of 3-Hz pacing, cells were superfused with $\mathrm{Na}^{+}-$and $\mathrm{Ca}^{2+}-$ free solution. Application of tetracaine $(1 \mathrm{mM})$ reduced the baseline fluorescence (leak). Caffeine (Caff; $10 \mathrm{mM})$ was applied at the end of the protocol to assess the $\mathrm{SR} \mathrm{Ca} \mathrm{Ca}^{2+}$ load. The box made of dashed lines indicates the region expanded in E. (E) Representative signals during tetracaine application from WT, RyR2-S2808D+/+, and S107-treated RyR2-S2808D+/+ myocytes. (F) Pooled data from the number of cells indicated parenthetically, showing the mean \pm SEM leak/load relationship of WT and RyR2-S2808D+/+ myocytes in the presence and absence of S107 (1 $\mu$ M). Values represent the magnitude of reduction due to tetracaine expressed as a percentage of the increase in signal in response to caffeine $\left({ }^{*} P<0.05\right)$. Myocytes were prepared from 5 or 6 mice in each experimental group.

of calstabin2, JTV-519 is also a multichannel blocker (41). We therefore developed a more RyR specific, orally available 1,4-benzothiazepine derivative, $\mathrm{S} 107$, referred to herein as a rycal, which has minimal off-target activity, based on extensive testing (36). Addition of the rycal S107 $(1 \mu \mathrm{M})$ increased calstabin2 binding to PKA-phosphorylated WT RyR2 samples treated with $\mathrm{H}_{2} \mathrm{O}_{2}$ by approximately 2 fold (Figure 3D). Oxidation of RyR2 also caused depletion of PDE4D3 from the channel complex, providing a mechanism for the earlier observation that PDE4D3 is depleted from RyR2 complexes in failing hearts and contributes to the PKA hyperphosphorylation of RyR2 in HF (16) (Figure 3E). Stable and transient (but not inducible) heterologous expression of recombinant RyR2-S2808D channels resulted in oxidation of the channel, possibly due to intracellular $\mathrm{Ca}^{2+}$ leak, which poisons mitochondria and leads to ROS production (Figure $3 \mathrm{~F}$ ). This finding suggests that variation in binding of calstabin 2 to RyR2-S2808D channels expressed in heterologous systems may be explained by variations in the state of oxidation of the channel. Thus, both PKA phosphorylation and $\mathrm{H}_{2} \mathrm{O}_{2}$ alone reduce the binding of calstabin2 to RyR2, and the combination of PKA phosphorylation plus oxidation almost completely depletes calstabin2 from the RyR2 complex. Treatment with the rycal S107 partially inhibits the PKA/ $\mathrm{H}_{2} \mathrm{O}_{2}$-mediated depletion of calstabin2 from the RyR2 complex.

RyR2 channels isolated from RyR2-S2808D $D^{+/+}$mice are "leaky." RyR2 single-channel activity was recorded in the presence of low $\mathrm{Ca}^{2+}$ $(150 \mathrm{nM})$ in the cis (cytosolic) planar lipid bilayer chamber. This $\left[\mathrm{Ca}^{2+}\right]$ corresponds to cytosolic $\left[\mathrm{Ca}^{2+}\right]$ in cardiomyocytes during diastole, when RyR2 channels in normal, non-failing hearts are tightly closed to prevent a diastolic SR $\mathrm{Ca}^{2+}$ leak (42). Channel activity of RyR2 isolated from 6-week-old RyR2-S2808D mice was similar to channel activity in samples from age-matched WT mice, consistent with the presence of calstabin 2 on these channels. In contrast, calstabin2-depleted RyR2 channels from 10-month-old RyR2-S2808D ${ }^{+/+}$mice exhibited significantly increased open probability (Po) compared with channels from WT control mice (Po, $0.310 \pm 0.049[n=9]$ vs. $0.029 \pm 0.007[n=6]$ for RyR2-S2808D and WT channels, respectively; $P<0.001$ ) (Figure 4, A-C). Moreover, and in contrast to cardiac RyR2 channels from WT mice and 6-week-old RyR2-S2808D-/- mice (Figure 4D), channels from the 10 -month-old RyR2-S2808D $\mathrm{D}^{+/+}$mice exhibited subconductance states indicative of channels that are depleted of calstabin2 (Figure 4F and refs. 13 and 42).

The single channel results (Figure 4) and RyR2 complex remodeling (Figure 2) suggest that the mechanism underlying the progressive dysfunction in the RyR2-S2808D ${ }^{+/+}$mice is a diastolic SR $\mathrm{Ca}^{2+}$ leak, resulting from depletion of calstabin2 from the RyR2 complex, induced by mimicking chronic PKA phosphorylation at Ser2808, combined with oxidation and nitrosylation of the channel. In order to asses $\mathrm{Ca}^{2+}$ leak, we measured $\mathrm{Ca}^{2+}$ sparks in myocytes after termination of $3-\mathrm{Hz}$ field stimulation. Myocytes from 6- to 8-month-old RyR2-S2808D mice exhibited an increased spark frequency compared with that of myocytes from agematched control WT mice $\left(P<0.05\right.$ for WT vs. RyR2-S2808D ${ }^{+/+}$ cardiomyocytes; $n=27$ and 32, respectively; Figure 5, A and B). 

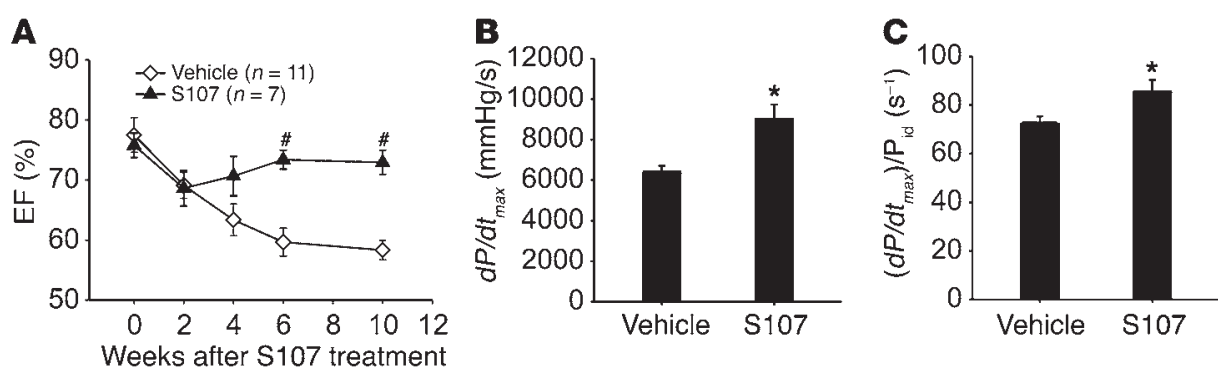

\section{Figure 6}

S107 improves cardiac function in RyR2-S2808D+/+ mice. (A) Echocardiographic measurements during a 10-week treatment period, showing that the $S 107$-treated $(20 \mathrm{mg} / \mathrm{kg} / \mathrm{d}$ via osmotic pump) group exhibited preserved cardiac function compared with the vehicle-treated group ( $P<0.01$ versus vehicle-treated group). (B and $\mathbf{C}$ ) Cardiac catheterization was performed at the end of study, and both $d P / d t_{\max }$ and $\left(d P / d t_{\max }\right) / P_{\text {id }}$, where $P_{\text {id }}$ indicates the instantaneous developed pressure in $\mathrm{mmHg}$ and $\left(\mathrm{s}^{-1}\right)$ is the unit of measure for $\left(d P / d t_{\max }\right) / \mathrm{P}_{\text {id }}$, showed a significant improvement in S107-treated group ( ${ }^{\star} P<0.05$ versus vehicle-treated group).

Sparks were also analyzed for amplitude, FWHM, and FDHM, and no significant differences in any of these parameters were observed. Caffeine responses were measured at the end of spark recording to assess $\mathrm{SR} \mathrm{Ca}^{2+}$ store content, since a diastolic SR $\mathrm{Ca}^{2+}$ leak would likely result in a reduced SR $\mathrm{Ca}^{2+}$ store. The amplitude of the caffeine-induced $\mathrm{Ca}^{2+}$ signal was significantly reduced in myocytes from RyR2-S2808D ${ }^{+/+}$mice after termination of $3-\mathrm{Hz}$ pacing, indicating a reduction in the $\mathrm{SR} \mathrm{Ca}^{2+}$ store content $\left(P<0.05\right.$ for WT vs. RyR2-S2808D ${ }^{+/+}$cardiomyocytes; $n=26$ and 40, respectively; Figure 5C).

We also determined the magnitude of $\mathrm{SR} \mathrm{Ca}^{2+}$ leak in myocytes using tetracaine treatment in the absence of extracellular $\mathrm{Na}^{+}$and $\mathrm{Ca}^{2+}$. This technique has been used to examine the extent of SR $\mathrm{Ca}^{2+}$ leak under various experimental conditions $(43,44)$. After the termination of field stimulation at $3 \mathrm{~Hz}$, extracellular $\mathrm{Na}^{+}$and $\mathrm{Ca}^{2+}$ were removed to eliminate plasmalemmal $\mathrm{Ca}^{2+}$ fluxes (Figure 5D). Tetracaine (1 mM) application, which blocks RyR2, caused a reduction in the baseline $\mathrm{Ca}^{2+}$ levels (Figure 5E) that was larger in RyR2-S2808D ${ }^{+/+}$myocytes compared with that in WT myocytes, indicating a significantly increased diastolic $\mathrm{Ca}^{2+}$ leak in the RyR2-S2808D ${ }^{+/+}$myocytes $\left(P<0.05\right.$ for WT vs. RyR2-S2808D ${ }^{+/+}$ cardiomyocytes; $n=44$ and 48 , respectively). The magnitude of this diastolic $\mathrm{Ca}^{2+}$ leak was expressed as a percentage of the caffeine releasable pool (Figure 5F).

Acute pretreatment of cardiomyocytes with the rycal S107 $(1 \mu \mathrm{M})$ for 2 hours completely eliminated the increased diastolic SR $\mathrm{Ca}^{2+}$ leak in RyR2-S2808D ${ }^{+/+}$myocytes $\left(P<0.05\right.$ for RyR2-S2808D ${ }^{+/+}$ cardiomyocytes with or without S107 treatment; $n=28$ and 19 , respectively; Figure 5, E and F). These results provide direct measurements of the effects of S107 in reducing diastolic SR Ca ${ }^{2+}$ leak in intact myocytes and suggest that reducing $\mathrm{Ca}^{2+}$ leak in RyR2S2808D ${ }^{+/+}$mice with S107 may prevent the cardiac dysfunction observed in these mice.

S107 protects against cardiomyopathy in RyR2-S2808D ${ }^{+/+}$mice. S107 $(20 \mathrm{mg} / \mathrm{kg} / \mathrm{d})$ or vehicle controls were administrated to RyR2$\mathrm{S} 2808 \mathrm{D}^{+/+}$mice, beginning at 6 weeks of age, before the onset of detectable cardiac dysfunction to determine whether calstabin 2 dissociation from the RyR2 channel complex contributes to the agedependent cardiomyopathy in RyR2-S2808D ${ }^{+/+}$mice. Ten weeks of treatment with S107 significantly preserved cardiac function compared with that of the vehicle-treated group, as assessed by echocardiography (EF, $73 \% \pm 4 \%$ in S107-treated group [ $n=7]$ vs. $58 \% \pm 4 \%$ in vehicle-treated group $[n=11] ; P<0.05)$ and hemodynamic measurements $\left(d P / d t_{\max }, 9,009 \pm 726 \mathrm{mmHg} / \mathrm{s}\right.$ in $\mathrm{S} 107$-treated group vs. $6,301 \pm 432 \mathrm{mmHg} / \mathrm{s}$ in vehicle-treated group; $P<0.05$ ) (Figure 6 and Supplemental Table 3). These results suggest that calstabin2 dissociation from RyR2 channels contributes to the development of cardiomyopathy in the RyR2-S2808D ${ }^{+/+}$mice.

Mimicking chronic PKA byperphosphorylation of RyR2 increases mortality after MI. We next sought to determine whether mimicking chronic PKA hyperphosphorylation of RyR2 affects mortality after MI, since we have previously reported that PKA hyperphosphorylation of RyR2 contributes to progression of ischemic $\operatorname{HF}(13,15)$. Homozygous RyR2-S2808D ${ }^{+/+}$mice (5-6 months old) and age- and gender-matched WT littermates were subjected to proximal left anterior descending coronary artery ligation to induce MI, which resulted in progressive HF (Figure 7A).

Echocardiographic measurements revealed more severe LV dysfunction and significantly increased cardiac dimensions in RyR2S2808D ${ }^{+/+}$mice $(n=10)$ as early as 1 week after MI, compared with WT littermates $(n=15)$ (Figure $7, \mathrm{~A}-\mathrm{D})$. However, at the earliest age at which it is technically feasible to perform MI surgery on mice ( 4 months), the RyR2-S2808D ${ }^{+/+}$mice already exhibited a mild decrease in cardiac function (the EF for WT mice was $\sim 80 \%$ and for RyR2-S2808D ${ }^{+/+}$mice was $\sim 60 \%$ at this age). EF at 1 week after MI was $38 \% \pm 3 \%$ in RyR2-S2808D ${ }^{+/+}$mice and $50 \% \pm 2 \%$ in WT littermates $(P<0.001)$, while $\mathrm{LV}$ end-diastolic diameter was $4.65 \pm 0.11 \mathrm{~mm}$ in RyR2-S2808 $\mathrm{D}^{+/+}$mice and $4.22 \pm 0.07 \mathrm{~mm}$ in WT littermates, respectively $(P<0.01)$ (Figure 7, B and C). At week 8 after MI, the cardiac function and LV size, as measured using echocardiography, showed comparable values between WT and RyR2-S2808D ${ }^{+/+}$mice (Figure 7, A-C). Infarct sizes at the end of the study ( 8 weeks after $\mathrm{MI}$ ) revealed no significant differences between WT $(33.8 \% \pm 5.2 \% \mathrm{LV}, n=15)$ and RyR2-S2808D ${ }^{+/+}$mice $(32.1 \% \pm 3.1 \% \mathrm{LV}, n=10)$. The RyR2-S2808D ${ }^{+/+}$mice exhibited more advanced HF progression immediately after MI, probably in part due to the fact that they already have mild cardiac dysfunction at the time of the MI. The WT mice "catch up" to the RyR2S2808D ${ }^{+/+}$mice in terms of HF progression, because they too develop PKA hyperphosphorylation of RyR2, and by 8 weeks after MI, their RyR2 complexes are depleted of calstabin2 just like those of the RyR2-S2808D ${ }^{+/+}$mice (for example, see Figure 8E).

We also compared the survival of the RyR2-S2808D mice $(n=30)$ to WT mice $(n=32)$ after MI (Supplemental Table 4$)$. The KaplanMeier survival curve showed significantly increased mortality in RyR2-S2808D ${ }^{+/+}$mice compared with WT mice, during a period of 
A
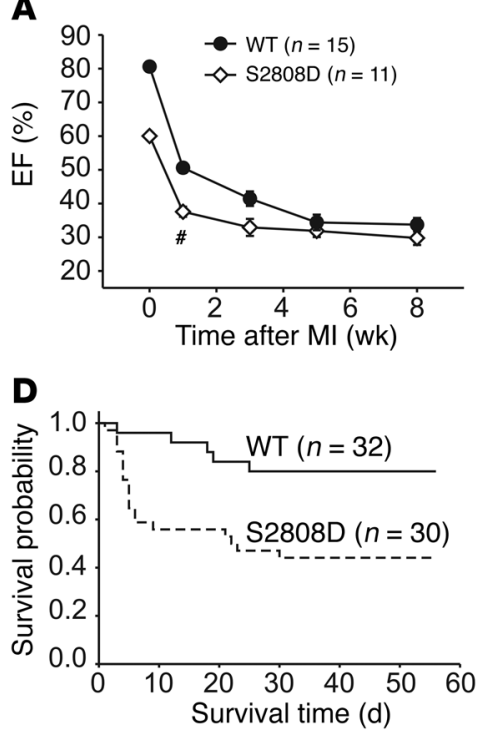

B
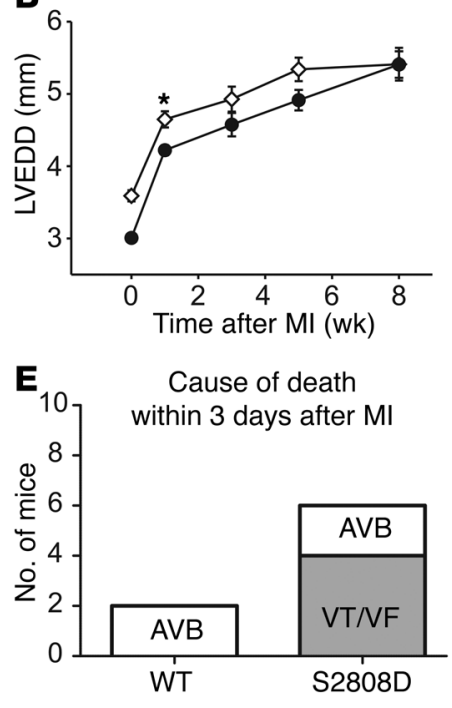

C

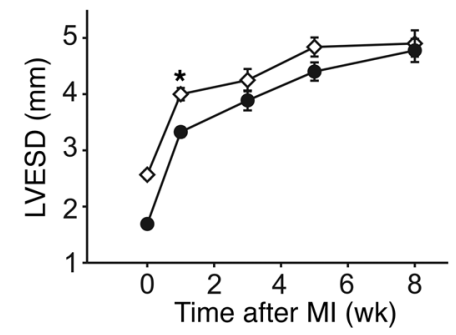

$\mathbf{F}$

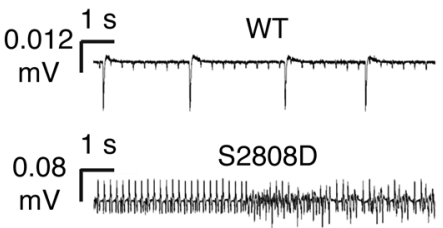

Figure 7

Increased mortality after MI in RyR2-S2808D+/+ mice. (A-C) Serial echocardiographic measurements after MI. (D) Kaplan-Meier survival curve of RyR2-S2808D ${ }^{+/+}$mice $(n=30)$ and WT littermates $(n=32)$ after Ml. The solid line represents WT mice, and the dashed line represents RyR2S2808D $+/+$ mice. (E) Telemeters were implanted in WT $(n=8)$ and RyR2-S2808D $(n=8)$ mice and cause of death within 3 days after MI was determined. AVB, atrial-ventricular block; VF, ventricular fibrillation; VT, ventricular tachycardia. (F) Representative telemetry traces depicting atrialventricular block in a WT mouse and ventricular tachycardia in a RyR2-S2808D ${ }^{+/+}$mouse after MI. ${ }^{\#} P<0.01$ versus WT; ${ }^{*} P<0.05$ versus WT.

up to 56 days after MI $(P<0.01)$. At 56 days after MI, the mortality rate for RyR2-S2808D ${ }^{+/+}$mice was $56 \%$ compared with $20 \%$ for WT mice, with most of the additional deaths occurring during the first week after MI (Figure 7D). Autopsies on the mice did not reveal any increased incidence in cardiac rupture or increased infarct size. The increased mortality in the RyR2-S2808D ${ }^{+/+}$mice was likely due to an increased incidence of sudden cardiac death within 7 days after MI secondary to ventricular arrhythmias observed in a separate group of mice (WT, $n=8$; RyR2-S2808D, $n=8$ ) using telemetric recordings (Figure $7, \mathrm{E}$ and $\mathrm{F}$ ). These data suggest that the presence of "leaky" RyR2-S2808D channels at the time of MI increases the risk for fatal ventricular arrhythmias.

Mimicking chronic RyR2 PKA phosphorylation reduces the efficacy of $\beta$-AR blockade in preventing HF progression. It is well established that $\beta$-blocker therapy improves cardiac function and reduces mortality in chronic HF both in humans and in animal models of $\operatorname{HF}(3,45)$. The mechanism underlying $\beta$-blocker therapy continues to be incompletely understood, despite recent data from our group $(5,6)$ and others $(20)$ showing that $\beta$-AR blockade indirectly inhibits PKA hyperphosphorylation of RyR2 and prevents calstabin2 depletion from the RyR2 complex in animal models of HF and in patients with HF. We reasoned that the RyR2-S2808D ${ }^{+/+}$mice might be relatively resistant to treatment of $\mathrm{HF}$ with $\beta$-blockers as compared with WT mice, because the RyR2-S2808D ${ }^{+/+}$mice are models for chronically PKA hyperphosphorylated RyR2 channels, yet blocking adrenergic signaling with $\beta$-blockers cannot alter the degree of PKA phosphorylation of the RyR2-S2808D ${ }^{+/+}$channels. To test this hypothesis the effects of S107 and the $\beta$-blockers metoprolol and carvedilol were compared in the treatment of ischemic HF in RyR2-S2808D ${ }^{+/+}$and WT mice. RyR2-S2808D ${ }^{+/+}$ and WT mice were subjected to acute MI and divided into 4 subgroups: vehicle, S107 (20 mg/kg/d), metoprolol $(30 \mathrm{mg} / \mathrm{kg} / \mathrm{d}$ and
$300 \mathrm{mg} / \mathrm{kg} / \mathrm{d}$ ), or carvedilol (10 mg/kg/d) treated. All drugs were administered via osmotic pumps. All drug treatments were started 1 week after MI, and cardiac function was evaluated by echocardiography at the 2 nd and 4 th weeks after commencing treatment (3rd and 5th weeks after MI). After 4 weeks of treatment with S107, metoprolol (high or low dose), or carvedilol, post-MI WT mice all exhibited preserved EF (Figure 8A and Supplemental Table 5) and systolic $\left(d P / d t_{\max }\right)$ function (Figure $\left.8 \mathrm{C}\right)$, compared with that of vehicle-treated animals.

In striking contrast to the WT mice, neither metoprolol (high or low dose) nor carvedilol prevented HF progression in post-MI RyR2-S2808D ${ }^{+/+}$mice, whereas S107 significantly prevented HF progression in these mice, as measured using both echocardiography and cardiac catheterization (Figure 8, B and D). Infarct sizes (measured by histologic cross sectional analyses) were not significantly different between vehicle-treated (35\% $\pm 4 \% \mathrm{LV})$, metoprolol-treated $(34 \% \pm 3 \% \mathrm{LV})$, and S107-treated (35\% $\pm 5 \% \mathrm{LV})$ RyR2-S2808D ${ }^{+/+}$mice. The lack of $\beta$-blocker efficacy in post-MI RyR2-S2808D ${ }^{+/+}$mice was not due to deficiencies in $\beta$-AR signaling, as radioligand binding results demonstrated similar $\beta$-AR receptor densities in 4-month-old WT and RyR2-S2808D ${ }^{+/+}$mice (Supplemental Table 6), and the ability of acute Iso treatment to stimulate phosphorylation of phospholamban at Ser 16 was equivalent in both groups (Supplemental Figure 4).

PKA hyperphosphorylation and depletion of calstabin 2 from the RyR2 complex were evident in post-MI vehicle-treated animals (Figure 8, E and F). Metoprolol treatment reduced PKA phosphorylation of RyR2 and reduced depletion of calstabin2 from the RyR2 complex in post-MI WT mice but not in post-MI RyR2-S20808D ${ }^{+/+}$mice (Figure 8, E and F). S107 did not prevent the PKA hyperphosphorylation of RyR2 in post-MI WT mice but did prevent depletion of calstabin 2 from the RyR 2 complex in 
A
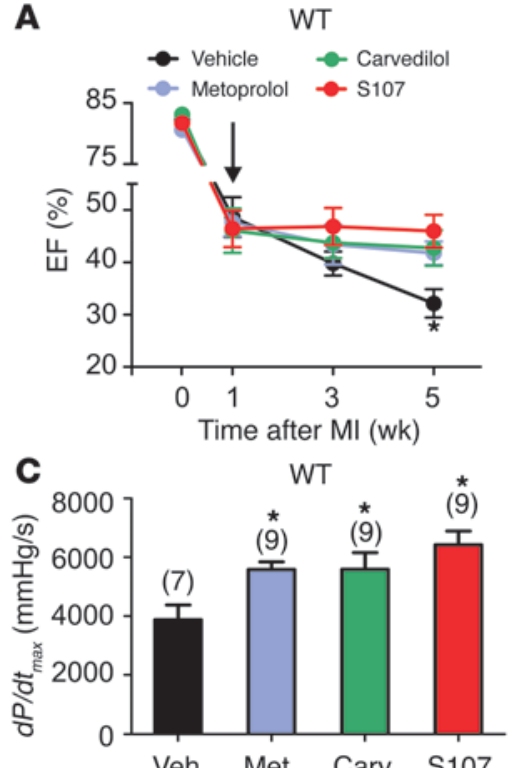

$\mathbf{E}$

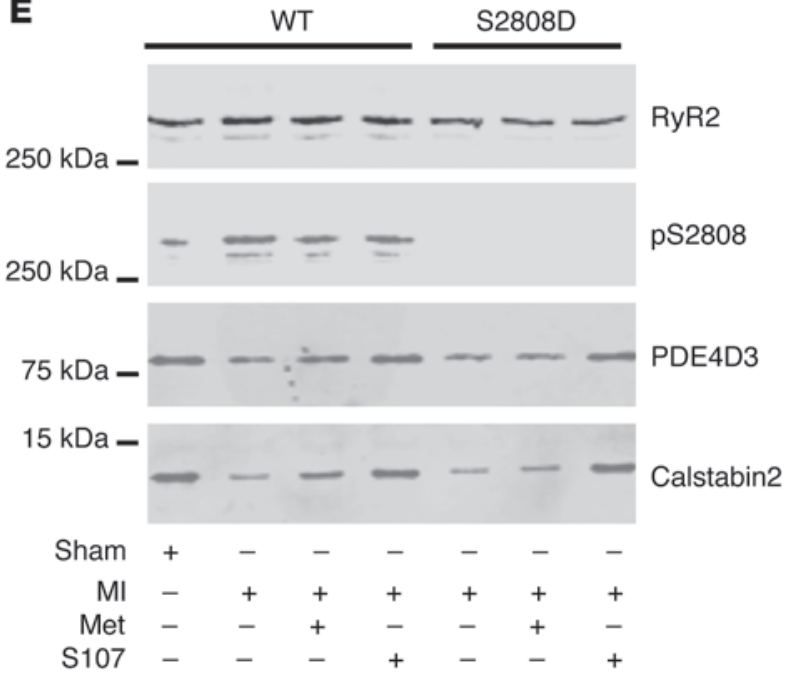

B
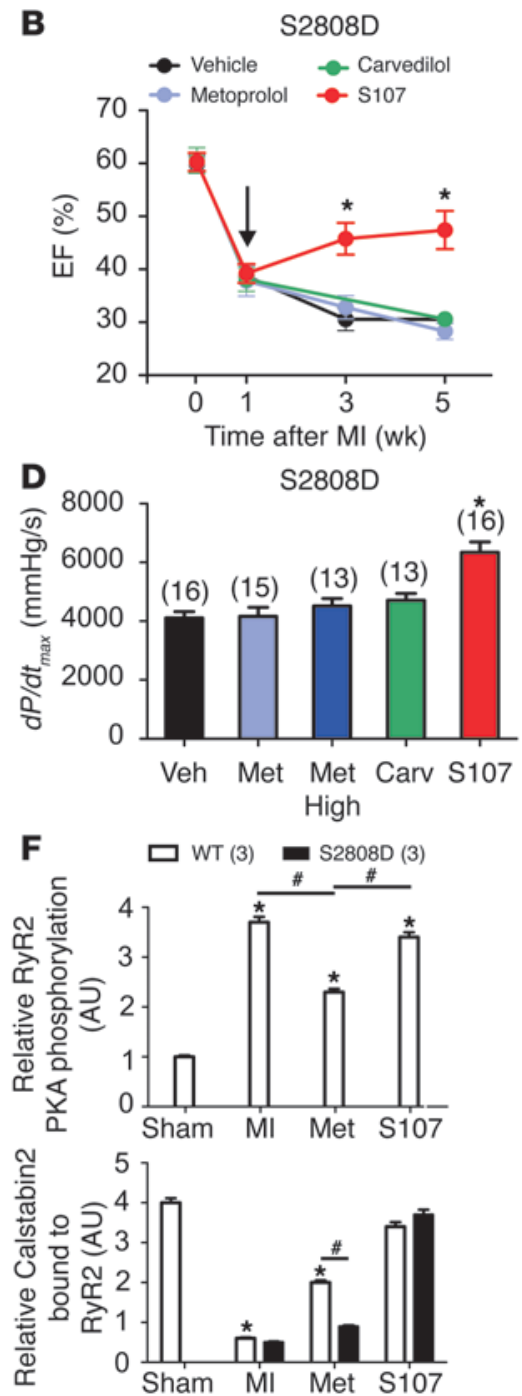

Figure 8

S107 but not metoprolol or carvedilol improves cardiac function in RyR-S2808D+/+ mice after MI. (A and B) Echocardiographic measurements at baseline, 1, 3, and 5 weeks after MI. Treatment started 1 week after MI (arrows). (A) In the WT group, S107, metoprolol (Met), and carvedilol (Carv) showed a beneficial effect at the end of study (week 4 of treatment). (B) In the RyR2-S2808D+/+ group, only S107 inhibited HF progression. ${ }^{*} P<0.05$ versus vehicle group. (C and D) Hemodynamic data were obtained by cardiac catheterization at the end of study. (C) In the WT group, S107, metoprolol, and carvedilol improved LV systolic function ( $\left.d P / d t_{\max }\right)$. (D) In the RyR2-S2808D ${ }^{+/+}$group, only S107 improved LV systolic function. The number of mice in each treatment group is indicated by the parenthetical numbers over each bar. Black represents vehicle; light blue represents low-dose metoprolol (30 mg/kg/d); dark blue represents high-dose metoprolol (300 mg/kg/d); green represents carvedilol $(10 \mathrm{mg} / \mathrm{kg} / \mathrm{d})$; and red represents $\mathrm{S} 107$ (30 mg/kg/d). ${ }^{*} P<0.05$ versus vehicle group. (E) Representative immunoblots from in vivo studies. Equivalent amounts of RyR2 were immunoprecipitated from cardiac lysates using an anti-RyR2 antibody. In the WT group, MI increased RyR2-S2808 PKA phosphorylation and calstabin2 depletion from the RyR2 channel complex and metoprolol decreased RyR2-S2808 PKA phosphorylation and reduced depletion of calstabin2 from the cardiac RyR2 channel complex. In the RyR2-S2808D+/+ group, calstabin2 was depleted from RyR2 channel complex in both vehicle- and metoprolol-treated groups, whereas S107 reduced depletion of calstabin2 from the cardiac RyR2 channel complex. (F) Pooled data from 3 separate immunoblots. ${ }^{*} P<0.05$ versus sham; ${ }^{*} P<0.05$ versus metoprolol treated.

both post-MI WT and post-MI RyR2-S20808D ${ }^{+/+}$mice treated with S107 (Figure 8, E and F).

In both WT and RyR2-S2808D ${ }^{+/+}$groups, all animals treated with metoprolol or carvedilol exhibited a significantly reduced heart rate compared with that of vehicle or S107 subgroups (Supplemental Figure 5). This is consistent with physiologic $\beta$-blockade and provides evidence that the mice were treated with appropriate doses of metoprolol or carvedilol. Furthermore, RyR2-S20808D ${ }^{+/+}$mice treated with a higher dose of metoprolol were also not protected against HF progression (Figure 8, B and D). Taken together, these data show that preventing PKA phosphorylation of RyR2-Ser2808 is an important determinant of the efficacy of $\beta$-AR blockade treatment in HF. 


\section{Discussion}

Despite the fact that $\beta$-blockers are standard therapy in HF, there is no accepted understanding of their mechanism of action in HF. In fact, multiple mechanisms have been proposed, including increased $\beta$-AR density (46) and altered myocardial gene expression (47). Previously, we reported that systemic administration of $\beta$-blockers reduces PKA hyperphosphorylation of RyR2 and restores calstabin 2 binding to RyR2 channels in animal models of HF and in cardiac samples from human heart transplant recipients $(5,6)$. We interpreted these findings as indicating that in failing hearts $\beta$-blockers can indirectly fix the leak in RyR2 channels by inhibiting PKA phosphorylation of the channel. The present study markedly extends these earlier studies by showing that $\beta$-blockers can improve cardiac function after MI in WT but not in RyR2-S2808D ${ }^{+/+}$mice (Figure 8), indicating that inhibition of PKA hyperphosphorylation of RyR2 is a mechanism, which we believe to be novel, by which $\beta$-blockers improve cardiac function after MI. Since $\beta$-blockers are standard therapy for HF, new insights into their mechanism of action in this disorder should open the doors for developing novel therapeutic approaches designed to target $\beta$-AR-dependent pathways that affect diastolic SR Ca ${ }^{2+}$ leak via PKA hyperphosphorylated RyR2 channels in failing hearts.

One potential limitation of the current study is that the reduced cardiac function present in the RyR2-S2808D mice at the time they underwent MI and $\beta$-blocker treatment may have limited the therapeutic efficacy of the $\beta$-blocker treatment. Moreover, our study does not exclude the possibility that $\beta$-blockers could be providing beneficial effects after MI via additional mechanisms that are independent of their ability to inhibit PKA phosphorylation of the RyR2 channel. For example, since the RyR2-S2808D mutation in our mice is neither inducible nor tissue specific, the observed resistance to $\beta$-blockers after MI could be explained by developmental and/or extracardiac effects that we have not detected.

In addition to providing a potential mechanism for the action of $\beta$-blockers in HF, the present study elucidates the impact of chronic PKA hyperphosphorylation of RyR2 on cardiac function in the absence of the many other changes that occur in HF. Cardiac function in young RyR2-S2808D ${ }^{+/+}$mice was similar to that in WT mice but was impaired as the mutant mice aged. This suggests that a low level of SR $\mathrm{Ca}^{2+}$ leak caused by chronic phosphorylation of RyR2 promotes cardiac dysfunction over time. The finding that S107, which inhibits depletion of calstabin2 from the RyR2 macromolecular complex, rescued cardiac dysfunction indicates that loss of calstabin2 from the RyR2 macromolecular complex plays an important role in the development of the cardiomyopathy observed in adult RyR2-S2808D ${ }^{+/+}$mice. The finding that there was only modest $(-20 \%)$ depletion of calstabin 2 from RyR2-S2808D ${ }^{+/+}$channels in 6-week-old mice and near complete depletion of calstabin 2 from the RyR2 complexes in 1-year-old RyR2-S2808D ${ }^{+/+}$mice, combined with our earlier observations that recombinant RyR2-S2808D channels were depleted of calstabin2 $(48,49)$, suggested that other modifications of the RyR2 channel could combine with PKA phosphorylation to the cause depletion of calstabin2 from the channels. Indeed, we found that the oxidative state of the RyR2 channels influences the extent to which PKA phosphorylation of RyR2 causes depletion of calstabin2, as shown in Figure 2G. Thus, the progressive, age-dependent oxidation of RyR2 in the RyR2-S2808D ${ }^{+/+}$mice explains why there is an age-dependent depletion of calstabin2 from the RyR2S2808D channel complexes. In addition, in our experiments heterologously expressed recombinant RyR2-S2808D were also oxidized, possibly due to the intracellular $\mathrm{Ca}^{2+}$ leak, which can cause $\mathrm{Ca}^{2+}$ overload of mitochondria, resulting in production of ROS. This explains why these recombinant RyR2-S2808D channels are depleted of calstabin2 $(48,49)$.

It is likely that the current observation of the role of oxidation in determining the degree to which PKA phosphorylation of RyR2 causes depletion of calstabin2 from the channel explains the variability in the results of other groups who have tried to reproduce our findings $(29,50)$, because the oxidation of RyR2 was not known to influence the PKA phosphorylation-induced dissociation of calstabin2 from the channel. Moreover, given that oxidation and nitrosylation of RyR2, combined with PKA hyperphosphorylation of the channel, contribute to calstabin2 depletion from the RyR2 macromolecular complex, it is possible that different preparations of RyR2 may be nitrosylated or oxidized to varying degrees and this could contribute to discrepancies between assessments of the amounts of calstabin2 in RyR2 complexes from failing hearts or from heterologously expressed channels. Our present results support this idea, since the RyR2S2808D channels in the RyR2-S2808D ${ }^{+/+}$mice were not completely depleted of calstabin2 until they were also significantly oxidized and nitrosylated.

Using permeabilized cardiomyocytes, Bers and colleagues recently reported that calstabin 2 was bound to less than $20 \%$ of RyR2 (27). As a result of this finding, they suggested that calstabin2 depletion from RyR2 was unlikely to have a major effect on cardiac function. However, this low occupancy by calstabin 2 of its binding site in RyR2 is at odds with that reported by Fleischer and colleagues (51), who used radioligand binding to estimate that $83 \%$ of the calstabin 2 binding sites on RyR 2 were occupied in canine cardiac tissue. The large discrepancy between these 2 findings could be due to the fact that Bers et al. used permeabilized cardiomyocytes. Bers et al. also reported that PKA treatment did not disrupt calstabin2-RyR2 interactions in permeabilized myocytes, which agrees with our current binding experiments in which PKA phosphorylation of nonoxidized RyR2 alone did not cause complete calstabin2 depletion.

Our current results provide evidence for a possible link between RyR2-mediated leak and oxidative stress in cardiomyocytes. One potential mechanism is that a low level of $\mathrm{Ca}^{2+}$ leak via the RyR2S2808D channels triggers the production of reactive oxygen and nitrogen species via mitochondrial or NOS sources. Another possibility is that a conformational change in RyR2 is induced by the S2808D mutation that results in the exposure of residues susceptible to oxidative and/or nitrosative modification.

The combination of the S2808D mutation and oxidative/nitrosative changes could also trigger conformational changes in RyR2 that destabilize interactions with other regulatory proteins, including mAKAP, PDE4D3, PP1, and PP2A. This is supported by the $\mathrm{H}_{2} \mathrm{O}_{2}$-induced loss of PDE4D3 from RyR2 (Figure 3E). Based on earlier findings using recombinant channels, we expected that the S2808D mutation alone would be sufficient to disrupt channel activity and cause cardiac dysfunction. The present study demonstrates that it is likely the combined effect of the loss of calstabin 2 and other regulatory proteins that ultimately causes the cardiomyopathy observed in the RyR2-S2808D mice. Nevertheless, the finding that S107, which promotes calstabin2 binding to 
phosphorylated and oxidized RyR2, prevents the cardiomyopathy highlights the importance of preserving the binding of calstabin2 to RyR2 as a therapeutic strategy.

Taken together, our present findings that RyR2 channels are oxidized and nitrosylated in RyR2-S2808D ${ }^{+/+}$mice and in murine and human failing hearts with WT channels, combined with our previous studies showing that RyR1 channels are nitrosylated in skeletal muscle from WT mice subjected to extreme exercise and from a mouse model of Duchenne muscular dystrophy ( $m d x$ mice), provide support for a paradigm in which chronic stress, whether it be due to HF, muscular dystrophy, extreme prolonged exercise, or presumably other disorders, results in oxidation and nitrosylation of RyR1 and RyR2 channels, causing an intracellular $\mathrm{Ca}^{2+}$ leak, which can contribute to disease progression and further promote oxidation due to mitochondrial dysfunction secondary to calcium overload. The concept that RyR channels are regulated by oxidation during stress is consistent with data from a number of laboratories showing that these channels are sensitive to oxidation $(35,52-54)$.

Additional information about the role of PKA phosphorylation of RyR2 in HF progression comes from our experiments showing increased mortality after MI in RyR2-S2808D ${ }^{+/+}$mice (Figure 7). In these studies, the RyR2-S2808D ${ }^{+/+}$mice developed worse cardiac function in the first 2 weeks following MI (Figure 7A). In part, this was likely due to the fact that RyR2-S2808D ${ }^{+/+}$mice start with a slightly lower EF due to their cardiomyopathy, but the WT mice eventually "catch up" and exhibit a similar degree of cardiac dysfunction by 4-6 weeks after MI. The fact that the WT mice develop a similar degree of dysfunction as the RyR2$\mathrm{S} 2808 \mathrm{D}^{+/+}$mice is consistent with our findings of progressive PKA hyperphosphorylation of RyR2 after MI (13). In other words, by 4-6 weeks after MI the cardiac RyR2 channels are PKA hyperphosphorylated in the WT mice, rendering them equivalent to the RyR2-S2808D channels in terms of diastolic SR Ca ${ }^{2+}$ leak.

Other groups have challenged the importance of PKA phosphorylation of RyR2 in HF progression using a RyR2-S2808A knockin mouse (31). We showed that HF progression was significantly blunted in RyR2-S2808 $\mathrm{A}^{+/+}$mice harboring RyR2 channels that cannot be PKA phosphorylated (15), whereas Benkusky et al. reported that their RyR2-S2808 $\mathrm{A}^{+/+}$mice were not protected against HF progression (31). One difference was that we used a post-MI HF model, and they used a hypertrophy model that resulted in mild cardiac dysfunction 11 weeks after aortic banding. Nevertheless, their data actually support ours, as they show that the RyR2-S2808A mutation protects against cardiac dysfunction (in agreement with our findings but contrary to the title and abstract of their paper) (31). Indeed, Benkusky et al. reported that their RyR2-S2808A ${ }^{+/+}$mice had a preserved fractional shortening of $51.9 \% \pm 5.1 \%$ at 11 weeks after banding compared with a significantly reduced fractional shortening of $42.6 \% \pm 1.2 \%$ in WT mice, $P<0.05$ (31), as shown in their Supplemental Table 1.

In conclusion, we have used a mouse model mimicking chronic PKA hyperphosphorylation of RyR2 to address the mechanism by which $\beta$-blockers improve cardiac function in HF. Our data support a mechanism which we believe to be novel for $\beta$-blockers in HF that involves indirectly inhibiting PKA phosphorylation of RyR2. In addition, the RyR2-S2808D ${ }^{+/+}$model helps clarify some discrepancies in the literature regarding the role of PKA hyperphosphorylation in HF. Specifically, PKA phosphorylation of oxidized and/or nitrosylated RyR2 causes depletion of calstabin2 from the RyR2 complex promoting HF progression.

\section{Methods}

Surgical and animal procedures. RyR2-S2808D mice and age-matched WT littermates were maintained and studied according to protocols approved by the Institutional Animal Care and Use Committee of Columbia University. Mice were randomized to undergo either MI or a sham procedure. Ligation of the left anterior descending artery was performed as previous described (39). At different time points after MI, mice were anesthetized with $1.0 \%-1.5 \%$ isoflurane in $\mathrm{O}_{2}$, placed on a heating pad $\left(37^{\circ} \mathrm{C}\right)$, and cardiac function was assessed by echocardiography, using a Visualsonic Vevo 770 ultrasound equipped with a $30-\mathrm{MHz}$ transducer applied to the chest wall. Cardiac ventricular dimensions and $\mathrm{EF}$ were assessed in the $2 \mathrm{D}$ mode. Cardiac catheterization was used to assess contractility using a 1.4F high-fidelity micromanometer catheter (Millar Instruments) advanced via the right carotid artery into the LV (39). The IOX data acquisition system was used to analyze pressure-volume relationships (Emka Technologies). Analyses were performed by observers blinded to the genotype and/or treatment of the animals. To monitor cardiac rhythm after MI, radio transmitters (Data Sciences International) for ECG recording were implanted in mice before MI, and ECGs were recorded continuously for 7 days after MI.

S107 treatment. S107 was administered using osmotic pumps at a dose of $20 \mathrm{mg} / \mathrm{kg} / \mathrm{d}$ (Alzet Model 2004, $200 \mu \mathrm{l}$ total volume, $0.25 \mu \mathrm{l} / \mathrm{h}$ delivery, or Alzet Model 1004, $100 \mu \mathrm{l}$ total volume, $0.11 \mu \mathrm{l} / \mathrm{h}$ delivery; Durect), filled with either $200 \mu \mathrm{l}$ or $100 \mu \mathrm{l}$ of PBS or $200 \mu \mathrm{l}$ or $100 \mu \mathrm{l}$ of $\mathrm{S} 107(100 \mu \mathrm{g} / \mu \mathrm{l}$ for model 2004 and $200 \mu \mathrm{g} / \mu \mathrm{l}$ for model 1004 diluted in PBS) implanted subcutaneously on the dorsal surface using a horizontal incision at the neck. Standard food and water were provided ad libitum throughout the experiment.

Immunoprecipitation and immunoblot analyses. Cardiac homogenates $(100 \mu \mathrm{g})$ were used to immunoprecipitate RyR2 channels with anti-RyR antibody (55). All human studies were performed according to protocols approved by the Institutional Review Board of Columbia University Medical Center, and the heart samples were acquired as previously described; human study participants provided informed consent (6). RyR2 was immunoprecipitated from heart homogenate $(100 \mu \mathrm{g})$ with anti-RyR antibody ( $2 \mu 15029 \mathrm{Ab})$ in $0.5 \mathrm{ml}$ of a modified RIPA buffer (50 mM Tris-HCl, pH 7.4, 0.9\% NaCl, $5.0 \mathrm{mM} \mathrm{NaF}, 1.0 \mathrm{mM} \mathrm{Na}_{3} \mathrm{VO}_{4}$, $0.5 \%$ Triton-X100, and protease inhibitors) for 2 hours at $4{ }^{\circ} \mathrm{C}$. The samples were incubated with protein A sepharose beads (Amersham Pharmacia Biotech) at $4^{\circ} \mathrm{C}$ for 1 hour and washed 5 times with $1.0 \mathrm{ml}$ RIPA. Samples were heated to $95^{\circ} \mathrm{C}$ and size fractionated by PAGE $(6 \%$ for RyR, $15 \%$ for calstabin2). Proteins were transferred to nitrocellulose membranes and immunoblots were developed using the following antibodies: anti-calstabin $(1: 1,000)(55)$, anti-RyR $(5029 ; 1: 3,000)(55)$, anti-phospho-RyR2-pSer ${ }^{2808}(1: 5,000)(49)$, anti-PDE4D3 (1:1,000) (16), anti-PP2a (1:1,000) (BD Transduction Laboratories), anti-PP1 $(1: 1,000)$ (BD Transduction Laboratories), and anti-Cys-NO (1:1,000) (SigmaAldrich). Levels of RyR2 bound proteins were normalized to the total RyR2 immunoprecipitated (arbitrary units). All immunoblots were developed with the Odyssey system (LI-COR Inc.), using IR-labeled antimouse and anti-rabbit IgG (1:10,000 dilution) secondary antibodies.

For oxidation reactions, the cardiac SR (CSR) $(200 \mu \mathrm{g})$ was resuspended in buffer $(10 \mathrm{mM}$ Tris- $\mathrm{HCl}, 150 \mathrm{mM} \mathrm{NaCl}, \mathrm{pH} 7.2$, with complete protease inhibitors; Roche Applied Science) and was incubated with $1 \mathrm{mM}$ $\mathrm{H}_{2} \mathrm{O}_{2}$ for 30 minutes at room temperature. After reaction, the samples were centrifuged for 10 minutes at $100,000 \mathrm{~g}$, and the resulting pellet was washed with $200 \mu \mathrm{l}$ wash buffer $(10 \mathrm{mM}$ imidazole, $300 \mathrm{mM}$ sucrose, $\mathrm{pH}$ 7.4) and resuspended $50 \mu \mathrm{l}$ of buffer. To determine the effect of $\mathrm{H}_{2} \mathrm{O}_{2}$ treatment of the CSR on the RyR2 complex, $50 \mu \mathrm{g}$ of the treated CSR was immunoprecipitated with RyR antibody, and the immunoprecipitates were analyzed for RyR, PDE4D3, PP2A, PP1, and calstabin2 by Western analysis as described above. 
RyR2 oxidation. To detect RyR2 protein oxidation, isolated SR membrane samples $(50 \mu \mathrm{g})$ were immunoprecipitated as described above. Immunoprecipitates were treated with 2, 4-dinitrophenyl (DNP) hydrazine and the DNP-derivatized carbonyls were detected using an OxyBlot Protein Oxidation Detection Kit (catalog S7150, Chemicon International Inc.). Proteins were separated on $6 \%$ SDS-PAGE gels and transferred onto nitrocellulose membranes, and immunoblots were developed with an antiRyR antibody (Affinity Bioreagents, 1:2,000). The DNP signal associated with RyR was determined using an anti-DNP antibody $(1: 2,000)$.

Calstabin binding assay. CSR $(200 \mu \mathrm{g})$ was incubated with 40 units of PKA (Sigma-Aldrich) in a kinase buffer (50 mM Tris/PIPES, $8 \mathrm{mM} \mathrm{MgCl}_{2}$, 10 mM EGTA, pH 7.0). PKA phosphorylation was initiated by the addition of $100 \mu \mathrm{M} \mathrm{Mg}$-ATP, and the reactions were incubated for 30 minutes at $30^{\circ} \mathrm{C}$. After reaction, the samples were centrifuged for 10 minutes at $100,000 \mathrm{~g}$, and the resulting pellets were washed 2 times with $200 \mu \mathrm{l}$ wash buffer (10 mM imidazole, $300 \mathrm{mM}$ sucrose, $\mathrm{pH}$ 7.4).

For oxidation reactions, the CSR $(200 \mu \mathrm{g})$ was resuspended in buffer $(10 \mathrm{mM}$ Tris- $\mathrm{HCl}, 150 \mathrm{mM} \mathrm{NaCl}, \mathrm{pH} 7.2$, with Complete protease inhibitors (Roche Applied Science). Samples were incubated with $1 \mathrm{mM} \mathrm{H}_{2} \mathrm{O}_{2}$ for 30 minutes at room temperature. After reaction, the samples were centrifuged for 10 minutes at $100,000 \mathrm{~g}$, and the resulting pellet was washed 1 time with $200 \mu \mathrm{l}$ wash buffer (10 mM imidazole, $300 \mathrm{mM}$ sucrose, pH 7.4). Fifty $\mu \mathrm{g}$ of the treated CSR was immunoprecipitated with RyR antibody as described above. The immunoprecipitate was separated by PAGE, and immunoblots were developed using antibodies against RyR (Affinity Bioreagents, 1:2,000), PDE (1:2,000), and PP2A (1:1,000).

To determine calstabin binding, CSR samples (control, PKA treated, $\mathrm{H}_{2} \mathrm{O}_{2}$ treated, and PKA/ $\mathrm{H}_{2} \mathrm{O}_{2}$ treated) were resuspended in $200 \mu \mathrm{l}$ binding buffer (10 mM imidazole, $300 \mathrm{mM}$ sucrose, $150 \mathrm{mM} \mathrm{NaCl}$, pH 7.2). Calstabin binding reaction was initiated by addition of $\left[{ }^{35} \mathrm{~S}\right]$-calstabin $2(100 \mathrm{nM}$ final concentration). S107 $(1 \mu \mathrm{M})$ was also tested in a set of PKA $/ \mathrm{H}_{2} \mathrm{O}_{2}$-treated CSR. Samples were incubated at room temperature for 1 hour in binding buffer. Reaction was stopped by addition of ice-cold binding buffer and then filtered through GF/B Whatman filters pre-equilibrated with $0.015 \%$ PE. Filters were washed 3 times with $5 \mathrm{ml}$ of wash buffer (10 mM MOPS, $200 \mathrm{mM} \mathrm{NaCl}, \mathrm{pH}$ 7.4), dried, and counted. Specific counts were determined by subtracting the signal in which $10 \mu \mathrm{M}$ rapamycin was present.

Oxidative stress assessments. Hearts were homogenized in 5\% sulfosalicylic acid. The tissue homogenates were centrifuged for 10 minutes at $10,000 \mathrm{~g}$. Supernatant was stored at $4^{\circ} \mathrm{C}$ until assayed. The concentration of total GSH (GSSG + GSH) was measured in the homogenates using the GSH reductase $/ 5,5$ '-dithiobis-(2-nitrobenzoic acid) (DTNB) recycling assay (catalog GT35, Oxford Biomedical Research). The rate of DTNB formation was followed at $412 \mathrm{~nm}$ and is proportional to the sum of GSH and GSSG present. Reduced GSH values were calculated as the difference between total (GSSG + GSH) and oxidized (GSSG) GSH (assessed in the presence of a free thiol scavenger provided by the manufacturer) concentrations.

$\beta$-Blocker treatment. The dose of metoprolol $(30 \mathrm{mg} / \mathrm{kg} / \mathrm{d}$ in the low-dose group or $300 \mathrm{mg} / \mathrm{kg} / \mathrm{d}$ in the high-dose group) for HF therapy was chosen based on data from rodents (45). Metoprolol was delivered using the osmotic pump in a solution of PBS. Carvedilol $(10 \mathrm{mg} / \mathrm{kg} / \mathrm{d})$ was delivered using the osmotic pump in polyethylene glycol/distilled water (3:2 [vol/vol]).

Single-channel recordings. SR vesicles were prepared from pooled hearts from WT and RyR2-S2808D mice. Planar lipid bilayers were formed by painting a mixture of phosphatidylethanolamine and phosphatidylcholine $3: 1$ ratio; Avanti Polar Lipids) across a $200-\mu \mathrm{m}$ aperture in a polysulfonate cup (Warner Instruments) separating 2 chambers. The trans chamber $(1.0 \mathrm{ml})$, representing the intra-SR (luminal) compartment, was connected to the headstage input of a bilayer voltage clamp amplifier. The cis chamber $(1.0 \mathrm{ml})$, representing the cytoplasmic compartment, was held at virtual ground. The chamber solutions were as follows: 1 mM EGTA, 250:125 mM HEPES/ Tris, $50 \mathrm{mM} \mathrm{KCl}, 0.64 \mathrm{mM} \mathrm{CaCl}_{2}, \mathrm{pH} 7.35$, for cis solution; and $53 \mathrm{mM}$ $\mathrm{Ba}(\mathrm{OH})_{2}, 50 \mathrm{mM} \mathrm{KCl}, 250 \mathrm{mM}$ HEPES, pH 7.35, for trans solution. Single RyR2 channels were reconstituted by spontaneously fusing vesicles into the planar lipid bilayer, and after incorporation, the RyR2 channel activity was measured at $0 \mathrm{mV}$. At the end of each experiment, $5 \mu \mathrm{M}$ ryanodine was added to confirm RyR2 channel identity.

Intracellular calcium measurements. Ventricular cardiomyocytes isolated from WT and RyR2-S2808D ${ }^{+/+}$mice were loaded with the fluorescent $\mathrm{Ca}^{2+}$ indicator Fluo4-AM $(5 \mu \mathrm{M})$, and $\mathrm{Ca}^{2+}$ signals were recorded by exciting the dye with the 488-nm laser line from an argon laser. Fluorescence was collected through a $63 \mathrm{x} 1.4$ NA objective (Zeiss) and recorded using a Zeiss 5 Live LSM system. Line scans were collected at a rate of 500 lines per second. Myocytes were continually superfused with a Tyrodes solution composed of $140 \mathrm{mM} \mathrm{NaCl}, 5 \mathrm{mM} \mathrm{KCl}, 1 \mathrm{mM} \mathrm{MgCl} 2,1.8 \mathrm{mM} \mathrm{CaCl}_{2}, 10 \mathrm{mM}$ HEPES, $\mathrm{pH} 7.4$, with $\mathrm{NaOH}$. Myocytes were electrically paced at $3 \mathrm{~Hz}$ via platinum electrodes in the perfusion chamber using a MyoPacer (IonOptix). Calcium sparks were recorded for 2-3 seconds after the termination of pacing, and caffeine $(10 \mathrm{mM})$ was rapidly applied after spark recordings to evaluate SR load. Images were analyzed for sparks using the SparkMaster plug-in for ImageJ (http://rsbweb.nih.gov/ij/), and caffeine responses were normalized to baseline fluorescence $\left(\mathrm{F} / \mathrm{F}_{\mathrm{o}}\right)$ for comparison.

$\mathrm{Ca}^{2+}$ leak experiments. $\mathrm{Ca}^{2+}$ leak was assessed using tetracaine application in the absence of extracellular $\mathrm{Na}$ and $\mathrm{Ca}$. For these experiments, the $\mathrm{NaCl}$ was replaced with equimolar LiCl, EGTA $(0.5 \mathrm{mM})$ was added, and $\mathrm{pH} 7.4$ was established using $\mathrm{LiOH}$. Following the termination of $3-\mathrm{Hz}$ pacing, the myocytes were superfused with $\mathrm{Na}^{+}$- and $\mathrm{Ca}^{2+}$-free solutions, prior to the addition of $1 \mathrm{mM}$ tetracaine. Tetracaine was applied for 30 seconds prior to the addition of $10 \mathrm{mM}$ caffeine. The magnitude of the reduction in baseline due to tetracaine was expressed as a percentage of the caffeine response for leak comparisons between samples. S107 was applied (1 $\mu \mathrm{M})$ for 2 hours prior to leak measurements where indicated.

Statistics. Data are reported as mean \pm SEM unless indicated otherwise. Two-tailed Student's $t$ tests were used to compare means between groups. ANOVA was used when multiple groups were compared. $P<0.05$ was accepted as statistically significant.

\section{Acknowledgments}

This work was supported by a grant from the Fondation Leducq and NHLBI grants HL061503 and HL056180 (to A.R. Marks). M.J. Betzenhauser was supported by NHLBI Training grant HL087745.

Received for publication June 21, 2010, and accepted in revised form October 4, 2010.

Address correspondence to: Andrew R. Marks, Department of Physiology and Cellular Biophysics, Russ Berrie Medical Sciences Pavilion, Room 520, 1150, St. Nicholas Ave., New York, New York 10032, USA. Phone: 212.851.5337; Fax: 212.851.5346; E-mail: arm42@columbia.edu.
1. Lloyd-Jones D, et al. Heart disease and stroke statis tics--2010 update: a report from the American Heart Association. Circulation. 2010;121(7):e46-e215.

2. Colucci WS, et al. Carvedilol inhibits clinical progression in patients with mild symptoms of heart failure. US Carvedilol Heart Failure Study Group. Circulation. 1996;94(11):2800-2806

3. Packer M, et al. The effect of carvedilol on morbidity and mortality in patients with chronic heart failure. U.S. Carvedilol Heart Failure Study Group.
NEngl J Med. 1996;334(21):1349-1355.

4. Packer M, et al. Effect of carvedilol on survival in severe chronic heart failure. $N$ Engl J Med. 2001; 344(22):1651-1658.

5 . Reiken S, et al. beta-adrenergic receptor blockers 
restore cardiac calcium release channel (ryanodine receptor) structure and function in heart failure. Circulation. 2001;104(23):2843-2848.

6. Reiken S, et al. Beta-blockers restore calcium release channel function and improve cardiac muscle performance in human heart failure. Circulation. 2003;107(19):2459-2466.

7. Fabiato A. Calcium-induced release of calcium from the cardiac sarcoplasmic reticulum. Am J Physiol. 1983;245(1):C1-14.

8. Fabiato A. Simulated calcium current can both cause calcium loading in and trigger calcium release from the sarcoplasmic reticulum of a skinned canine cardiac Purkinje cell. J Gen Physiol. 1985;85(2):291-320.

9. Fabiato A, Fabiato F. Calcium and cardiac excitation-contraction coupling. Annu Rev Physiol. 1979;41:473-484.

10. Cannell MB, Cheng H, Lederer WJ. The control of calcium release in heart muscle. Science. 1995;268(5213):1045-1049.

11. Hasenfuss G, Pieske B. Calcium cycling in congestive heart failure. J Mol Cell Cardiol. 2002;34(8):951-969.

12. Chidsey CA, Braunwald E, Morrow AG, Mason DT. Myocardial norepinephrine concentration in man. Effects of reserpine and of congestive heart failure. N Engl J Med. 1963;269:653-658.

13. Marx SO, et al. PKA phosphorylation dissociates FKBP12.6 from the calcium release channel (ryanodine receptor): defective regulation in failing hearts. Cell. 2000;101(4):365-376.

14. Lehnart SE, et al. Leaky Ca2 + release channel/ryanodine receptor 2 causes seizures and sudden cardiac death in mice. J Clin Invest. 2008;118(6):2230-2245.

15. Wehrens XH, Lehnart SE, Reiken S, Vest JA, Wronska A, Marks AR. Ryanodine receptor / calcium release channel PKA phosphorylation: a critical mediator of heart failure progression. Proc Natl Acad Sci U S A. 2006;103(3):511-518.

16. Lehnart SE, et al. Phosphodiesterase 4D deficiency in the ryanodine-receptor complex promotes heart failure and arrhythmias. Cell. 2005;123(1):25-35.

17. Bers DM, Eisner DA, Valdivia HH. Sarcoplasmic reticulum $\mathrm{Ca} 2+$ and heart failure: roles of diastolic leak and Ca2+ transport. Circ Res. 2003;93(6):487-490.

18. Yano M, et al. Altered stoichiometry of FKBP12.6 versus ryanodine receptor as a cause of abnormal $\mathrm{Ca}(2+)$ leak through ryanodine receptor in heart failure. Circulation. 2000;102(17):2131-2136.

19. Antos CL, et al. Dilated cardiomyopathy and sudden death resulting from constitutive activation of protein kinase a. Circ Res. 2001;89(11):997-1004.

20. Doi M, et al. Propranolol prevents the development of heart failure by restoring FKBP12.6-mediated stabilization of ryanodine receptor. Circulation. 2002;105(11):1374-1379.

21. Yano M, et al. FKBP12.6-mediated stabilization of calcium-release channel (ryanodine receptor) as a novel therapeutic strategy against heart failure. Circulation. 2003;107(3):477-484.

22. Lindegger N, Niggli E. Paradoxical SR Ca2+ release in guinea-pig cardiac myocytes after beta-adrenergic stimulation revealed by two-photon photolysis of caged Ca2+. J Physiol. 2005;565(pt 3):801-813.

23. Obayashi M, et al. Spontaneous diastolic contractions and phosphorylation of the cardiac ryanodine receptor at serine-2808 in congestive heart failure in rat. Cardiovasc Res. 2006;69(1):140-151.

24. Ellison GM, et al. Acute beta-adrenergic overload produces myocyte damage through calcium leakage from the ryanodine receptor 2 but spares cardiac stem cells. J Biol Chem. 2007;282(15):11397-11409.

25. Chen-Izu Y, et al. Phosphorylation of RyR2 and shortening of RyR2 cluster spacing in spontaneously hypertensive rat with heart failure. $A m J$ Physiol Heart Circ Physiol. 2007;293(4):H2409-2417.

26. Cheng Y, et al. Bradycardic therapy improves left ventricular function and remodeling in dogs with coronary embolization-induced chronic heart failure. J Pharmacol Exp Ther. 2007;321(2):469-476.

27. Guo T, et al. Kinetics of FKBP12.6 binding to ryanodine receptors in permeabilized cardiac myocytes and effects on Ca sparks. Circ Res. 2010; 106(11):1743-1752

28. Jiang MT, Lokuta AJ, Farrell EF, Wolff MR, Haworth RA, Valdivia HH. Abnormal Ca2+release, but normal ryanodine receptors, in canine and human heart failure. Circ Res. 2002;91(11):1015-1022.

29. Xiao B, Sutherland C, Walsh MP, Chen SR. Protein kinase A phosphorylation at serine-2808 of the cardiac Ca2+-release channel (ryanodine receptor) does not dissociate 12.6-kDa FK506-binding protein (FKBP12.6). Circ Res. 2004;94(4):487-495.

30. Xiao B, et al. Characterization of a novel PKA phosphorylation site, serine-2030, reveals no PKA hyperphosphorylation of the cardiac ryanodine receptor in canine heart failure. Circ Res. 2005;96(8):847-855.

31. Benkusky NA, et al. Intact beta-adrenergic response and unmodified progression toward heart failure in mice with genetic ablation of a major protein kinase A phosphorylation site in the cardiac ryanodine receptor. Circ Res. 2007;101(8):819-829.

32. Shan J, et al. Phosphorylation of the ryanodine receptor mediates the cardiac fight or flight response in mice. J Clin Invest. 2010;120(12):4388-4398.

33. Belevych AE, et al. Redox modification of ryanodine receptors underlies calcium alternans in a canine model of sudden cardiac death. Cardiovas Res. 2009;84(3):387-395.

34. Terentyev D, et al. Redox modification of ryanodine receptors contributes to sarcoplasmic reticulum Ca2+ leak in chronic heart failure. Circ Res. 2008;103(12):1466-1472.

35. Aracena P, Tang W, Hamilton SL, Hidalgo C. Effects of S-glutathionylation and S-nitrosylation on calmodulin binding to triads and FKBP12 binding to type 1 calcium release channels. Antioxid Redox Signal. 2005;7(7-8):870-881.

36. Bellinger AM, et al. Remodeling of ryanodine receptor complex causes "leaky" channels: a molecular mechanism for decreased exercise capacity. Proc Natl Acad Sci U S A. 2008;105(6):2198-2202.

37. Fauconnier J, et al. Leaky RyR2 trigger ventricular arrhythmias in Duchenne muscular dystrophy. Proc Natl Acad Sci U S A. 2010;107(4):1559-1564.

38. Zissimopoulos S, Docrat N, Lai FA. Redox sensitivity of the ryanodine receptor interaction with FK506-binding protein. J Biol Chem. 2007;282(10):6976-6983.

39. Wehrens $\mathrm{XH}$, et al. Enhancing calstabin binding to ryanodine receptors improves cardiac and skeletal muscle function in heart failure. Proc Natl Acad Sci US A. 2005;102(27):9607-9612.

40. Wehrens XH, et al. Protection from cardiac arrhyth- mia through ryanodine receptor-stabilizing protein calstabin2. Science. 2004;304(5668):292-296

41. Hasumi H, Matsuda R, Shimamoto K, Hata Y, Kaneko N. K201, a multi-channel blocker, inhibits clofilium-induced torsades de pointes and attenuates an increase in repolarization. Eur J Pharmacol. 2007;555(1):54-60.

42. Wehrens XH, et al. FKBP12.6 deficiency and defective calcium release channel (ryanodine receptor) function linked to exercise-induced sudden cardiac death. Cell. 2003;113(7):829-840.

43. Curran J, Hinton MJ, Rios E, Bers DM, Shannon TR. Beta-adrenergic enhancement of sarcoplasmic reticulum calcium leak in cardiac myocytes is mediated by calcium/calmodulin-dependent protein kinase. Circ Res. 2007;100(3):391-398.

44. Shannon TR, Guo T, Bers DM. Ca2+ scraps: local depletions of free $[\mathrm{Ca} 2+]$ in cardiac sarcoplasmic reticulum during contractions leave substantial Ca2+ reserve. Circ Res. 2003;93(1):40-45.

45. Kanda T, Inoue M, Suzuki T, Murata K. Low-dose combination therapy with metoprolol and captopril for congestive heart failure in mice. Cardiovasc Drugs Ther. 1993;7(5):795-800.

46. Gilbert EM, et al. Comparative hemodynamic, left ventricular functional, and antiadrenergic effects of chronic treatment with metoprolol versus carvedilol in the failing heart. Circulation. 1996;94(11):2817-2825.

47. Lowes BD, et al. Myocardial gene expression in dilated cardiomyopathy treated with beta-blocking agents. N Engl J Med. 2002;346(18):1357-1365.

48. Huang F, Shan J, Reiken S, Wehrens XH, Marks AR. Analysis of calstabin2 (FKBP12.6)-ryanodine receptor interactions: rescue of heart failure by calstabin2 in mice. Proc Natl Acad Sci U S A. 2006; 103(9):3456-3461.

49. Wehrens XH, Lehnart SE, Reiken SR, Marks AR. $\mathrm{Ca} 2+/$ calmodulin-dependent protein kinase II phosphorylation regulates the cardiac ryanodine receptor. Circ Res. 2004;94(6):e61-70.

50. Stange M, Xu L, Balshaw D, Yamaguchi N, Meissner G. Characterization of recombinant skeletal muscle (Ser-2843) and cardiac muscle (Ser-2809) ryanodine receptor phosphorylation mutants. J Biol Chem. 2003;278(51):51693-51702.

51. Timerman AP, et al. Selective binding of FKBP12.6 by the cardiac ryanodine receptor. J Biol Chem. 1996; 271(34):20385-20391.

52. Aracena P, Sanchez G, Donoso P, Hamilton SL, Hidalgo C. S-glutathionylation decreases Mg2+ inhibition and S-nitrosylation enhances $\mathrm{Ca} 2+$ activation of RyR1 channels. J Biol Chem. 2003; 278(44):42927-42935.

53. Aracena-Parks P, Goonasekera SA, Gilman CP, Dirksen RT, Hidalgo C, Hamilton SL. Identification of cysteines involved in S-nitrosylation, S-glutathionylation, and oxidation to disulfides in ryanodine receptor type $1 . \mathrm{J} \mathrm{Biol} \mathrm{Chem.}$ 2006;281(52):40354-40368

54. Eu JP, Sun J, Xu L, Stamler JS, Meissner G. The skeletal muscle calcium release channel: coupled $\mathrm{O} 2$ sensor and NO signaling functions. Cell. 2000;102(4):499-509.

55. Jayaraman T, et al. FK506 binding protein associated with the calcium release channel (ryanodine receptor). J Biol Chem. 1992;267(14):9474-9477. 